\title{
On equilibrium Metropolis simulations on self-organized urban street networks
}

\author{
Jérôme G. M. Benoit ${ }^{1,2^{*}}$ (1) and Saif Eddin G. Jabari ${ }^{1,3}$
}

\author{
${ }^{*}$ Correspondence: \\ jerome.benoit@ronininstitute.org \\ 2 Ronin Institute, Montclair, NJ \\ 07043, USA \\ Full list of author information \\ is available at the end of the \\ article
}

\begin{abstract}
Urban street networks of unplanned or self-organized cities typically exhibit astonishing scale-free patterns. This scale-freeness can be shown, within the maximum entropy formalism (MaxEnt), as the manifestation of a fluctuating system that preserves on average some amount of information. Monte Carlo methods that can further this perspective are cruelly missing. Here we adapt to self-organized urban street networks the Metropolis algorithm. The "coming to equilibrium" distribution is established with MaxEnt by taking scale-freeness as prior hypothesis along with symmetry-conservation arguments. The equilibrium parameter is the scaling; its concomitant extensive quantity is, assuming our lack of knowledge, an amount of information. To design an ergodic dynamics, we disentangle the state-of-the-art street generating paradigms based on non-overlapping walks into layout-at-junction dynamics. Our adaptation reminisces the single-spin-flip Metropolis algorithm for Ising models. We thus expect Metropolis simulations to reveal that self-organized urban street networks, besides sustaining scale-freeness over a wide range of scalings, undergo a crossover as scaling varies - literature argues for a small-world crossover. Simulations for Central London are consistent against the state-of-the-art outputs over a realistic range of scaling exponents. Our illustrative Watts-Strogatz phase diagram with scaling as rewiring parameter demonstrates a small-world crossover curving within the realistic window 2-3; it also shows that the state-of-the-art outputs underlie relatively large worlds. Our Metropolis adaptation to self-organized urban street networks thusly appears as a scaling variant of the Watts-Strogatz model. Such insights may ultimately allow the urban profession to anticipate self-organization or unplanned evolution of urban street networks.
\end{abstract}

Keywords: Urban street networks, Self-organization, Scale-freeness, Metropolis algorithm, MaxEnt, Symmetries, Conserved quantities, Self-similarity, Surprisal, Graph matchings, Ising model, Watts-Strogatz model, Small-world crossover

\section{Introduction}

Unplanned or self-organized cities spontaneously undergo scaling coherences for which a comprehensive explanation is lacking (Rybski et al 2019). Scaling coherence, or scalefreeness, expresses apparent invariance under zooming-in or -out transformations. The scaling coherence of the spatial organization of a city is reflected in its streets: the streets of a self-organized city typically follow a scale-free behaviour which has attracted much attention from observational and theoretical researchers (Rosvall et al 2005; Porta et al author(s) and the source, provide a link to the Creative Commons licence, and indicate if changes were made. The images or other third party material in this article are included in the article's Creative Commons licence, unless indicated otherwise in a credit line to the material. If material is not included in the article's Creative Commons licence and your intended use is not permitted by statutory regulation or exceeds the permitted use, you will need to obtain permission directly from the copyright holder. To view a copy of this licence, visit http:// creativecommons.org/licenses/by/4.0/. 
2006; Crucitti et al 2006; Jiang et al 2008). We recently linked the scale-freeness of selforganized urban street networks to a preservation principle through a fluctuating mesoscopic model (Benoit and Jabari 2019a, b).

The invoked preservation principle is the Jaynes's Maximum Entropy principle (Jaynes 1957, 2003; Lawrence 2019). This principle assesses the most plausible probability distribution of a fluctuating system according to moment constraints. We inversely applied it by envisioning streets as mesoscopic objects governed by social interactions (Benoit and Jabari 2019a, b). We reflect the scaling coherence by randomly distributing their numbers of configurations according to a scale-free distribution, specifically, a discrete Pareto distribution (Clauset et al 2009). The discrete Pareto distribution results from a constraint on the first logarithm moment (Dover 2004). Since their configurations are equally probable due to our lack of knowledge, this constraint interprets itself as an information measure preservation. The predominance of a number of vital connections among social connections asymptotically leads to a discrete Pareto distribution for the number of junctions per street. We have what is observed among self-organized urban street networks. However promising the approach appears, we need to investigate it completely with some specific tools.

To study such fluctuating models, analytical and simulational methods are usually employed as complementary methods to obtain more complete and accurate interpretations. Our analytical framework is the maximum entropy formalism, a general formalism of modern probability theory partially inherited from statistical physics (Jaynes 2003; Grandy 1987; Lawrence 2019). For simulating fluctuating systems, physicists mostly rely on random sampling algorithms based on Markov chain Monte Carlo methods, often abbreviated as Monte Carlo methods (Newman and Barkema 1999; Landau and Binder 2015). Each thus-generated random sample enables us to obtain numerical results that we can confront to theoretical ones. The Monte Carlo method of first choice remains the algorithm pioneered by Nicolas Metropolis and his co-workers (Metropolis et al 1953; Newman and Barkema 1999).

Strictly speaking the Metropolis algorithm may apply to configurations of streets or their associated information networks. An information network (Rosvall et al 2005; Porta et al 2006) is a dual network representation of an urban street network that (i) associates each street to a node, and (ii) links each pair of nodes (streets) sharing a common junction (see Fig. 1 for illustration). It is this dual graph representation that reveals the underlying scale-freeness (Porta et al 2006; Crucitti et al 2006; Jiang et al 2008). For instance, the valence distribution of an information network associated to a self-organized urban street network typically follows a discrete Pareto distribution (Clauset et al 2009). This observed scale-freeness provides a clue to find the prior hypothesis (Jaynes 2003; Grandy 1987) necessary to construct a fluctuating mesoscopic model for the streets, that is, to model the probability distribution to which Monte Carlo simulations are "coming to equilibrium" (Newman and Barkema 1999; Landau and Binder 2015). For mimicking fluctuating transitions, we may use the property that one information network transforms into another when a junction alters its street layout.

Basically, a Monte Carlo simulation iterates a Markov process for generating a Markov chain of states, a sequence of states whose every state depends only on its predecessor 


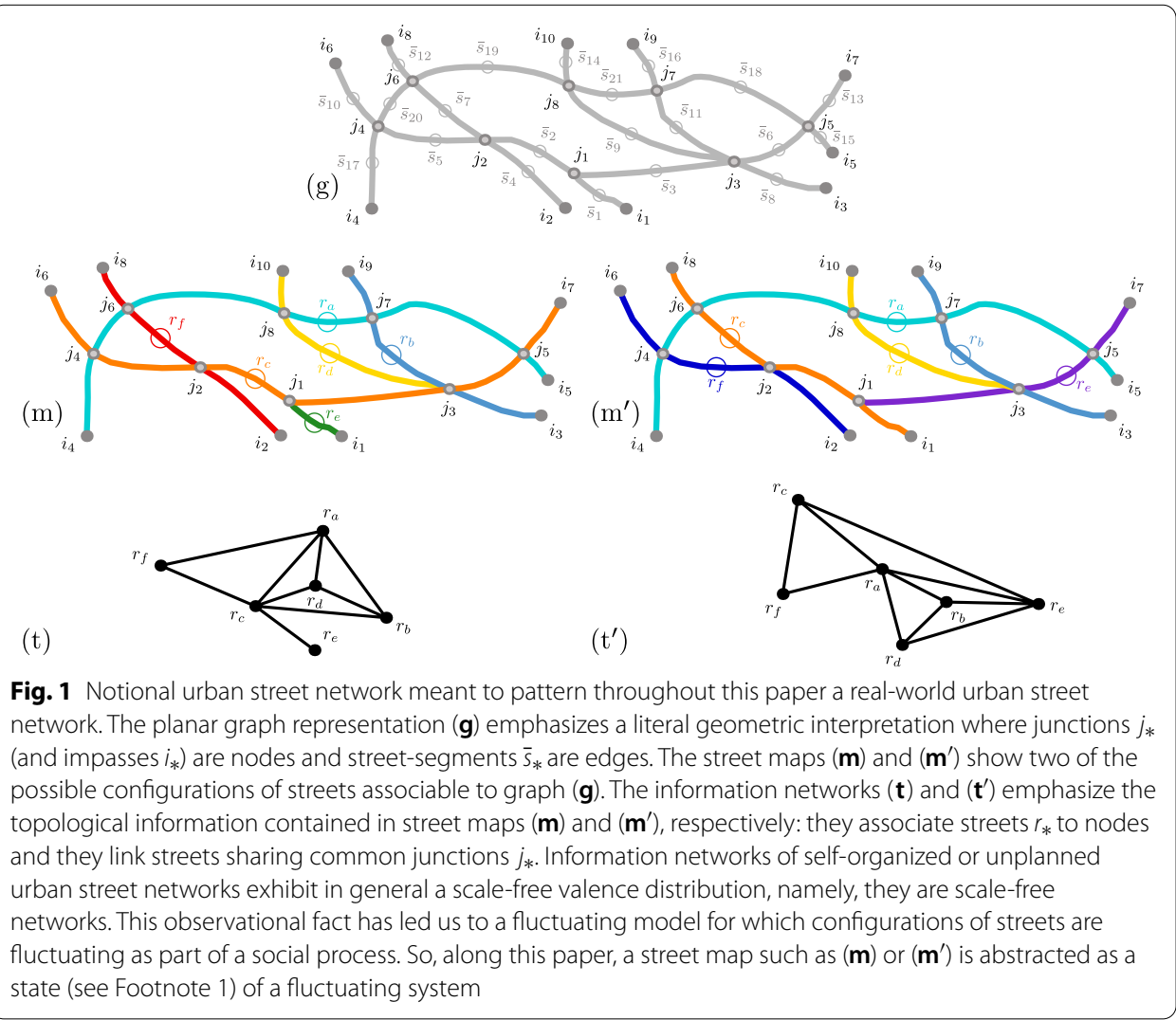

(Newman and Barkema 1999; Landau and Binder 2015). ${ }^{1}$ Here a state is any configuration of streets or its associated information network (see Fig. 1 for illustration). The Markov process is built so that the Markov chain reaches, when it is iterated enough times starting from any arbitrary state, the prescribed statistical equilibrium. To achieve this, the Markov process has to fulfil (i) the condition of detailed balance and (ii) the condition of ergodicity. The Metropolis algorithm is essentially an implementation choice for the former. The implementation of the condition of ergodicity relies on the details of the systems. The objective of this work is twofold. First, to present how a Metropolis algorithm adaptation can compel these two conditions for self-organized urban street networks. Second, to apprehend whether or not Metropolis simulations can provide pertinent "experimental" data to investigate their scaling coherence.

The rest of the paper presents our innovative modeling approach as follows. The second section carries out the two requested conditions. Firstly, once the probability distribution to come to equilibrium is established, the condition of detailed balance reduces to writing down the emblematic Metropolis acceptance ratio. Secondly, a short analysis enables us to disentangle the state-of-the-art paradigms for generating information networks into a constrained ergodic dynamics, which nonetheless recalls the classical

\footnotetext{
${ }^{1}$ A state is a set of quantities completely describing a system which does not include anything about its history. Along this notion, a dynamics is a map associating to a state another state which does not depend on the past states. A Markov process is a dynamics.
} 
single-spin-flip ergodic dynamics. This dynamics can potentially become unconstrained. Eventually, our Metropolis adaptation implements itself and compares easily against the classical single-spin-flip adaptation for Ising models. Next, the third section compares, over a wide range of scaling exponents, Metropolis generation series against state-ofthe-art outputs for Central London (United Kingdom). The range of consistency renders scaling investigations around their accepted scaling values feasible. As illustration, we plot the Watts-Strogatz phase diagram with scaling as rewiring parameter. We demonstrate thusly a small-world crossover curving at realistic scaling values. Accordingly the state-of-the-art outputs underlie relatively large worlds. In the concluding section, after a summary of the findings, we point how the presented methodology may contribute, as part of a fluctuating system approach, to change our perspective on urban street networks and, by extension, on cities.

\section{Implementation of the Metropolis algorithm}

This section shows how we can apply the classical Metropolis algorithm on unplanned or self-organized urban street networks to generate scale-free streets. We first adapt the most emblematic part, then we design two appropriate dynamics. Each dynamics aims to create from any current configuration of streets a new one. The emblematic part tells us whether or not to accept the new configurations of streets in order for their sequences to tend to a prescribed statistical equilibrium.

\section{The emblematic Metropolis acceptance ratio}

Typically Monte Carlo methods are applied to thermal systems. So applying them to a non-thermal system requires the extra preliminary work to frame the statistics of its steady fluctuations. The framework provided by the maximum entropy formalism allows us to derive an equilibrium distribution which is relevant to our scale-free system. This first achievement of our paper is necessary to implement any Monte Carlo method. The resulting Metropolis acceptance ratio takes a typical form.

\section{Scale-freeness as available information}

In the classic literature, the prescribed equilibrium distribution is de facto the Boltzmann distribution (Newman and Barkema 1999; Landau and Binder 2015). The same modern tools that derive the Boltzmann distribution from a conservation argument allows us to establish the prescribed equilibrium distribution of a scale-free system through a symmetry argument. We obtain a discrete Pareto distribution of an undefined quantity. This result should be folklore in some area, but we could not locate it in the literature.

At thermal equilibrium, the probability $p_{\mu}$ for a thermal system to occupy any state (see Footnote 1) $\mu$ is assumed to yield the Boltzmann distribution

$$
p_{\mu} \propto \mathrm{e}^{-\beta E_{\mu}}
$$

with $E_{\mu}$ the energy of state $\mu$ and $\beta$ the inverse temperature (Newman and Barkema 1999; Landau and Binder 2015; Grandy 1987). We have $\beta=1 / k T$ with $k$ the Boltzmann constant and $T$ the temperature. Nowadays the probability distribution (1) can easily be derived by applying the principle of maximum entropy (MAXENT) formulated by Jaynes 
(1957) as a general principle of probability theory (Jaynes 2003; Grandy 1987; Lawrence 2019). Within the maximum entropy formalism, Boltzmann probability (1) becomes the most plausible probability distribution that preserves the total energy of the system on average. This preservation is formally a constraint imposed on the mean of the energy. In practice the constraint is treated as a standard variational problem (Jaynes 2003; Grandy 1987) using the method of Lagrangian multipliers (see for example Applebaum 2008, App. 2). The Lagrangian writes (Jaynes 2003)

$$
\mathcal{L}_{\mathrm{TE}}\left(\left\{p_{\mu}\right\} ; v, \beta\right)=-\sum_{\mu} p_{\mu} \ln p_{\mu}-v\left[\sum_{\mu} p_{\mu}-1\right]-\beta\left[\sum_{\mu} p_{\mu} E_{\mu}-\langle E\rangle\right]
$$

where $p_{\mu}$ is our unknown probability distribution and the first Lagrange multiplier $v$ forces its normalization, while the second Lagrange multiplier $\beta$ imposes the mean energy to have the constant energy value $\langle E\rangle$. The stationary solution of Lagrangian (2) is the desired probability distribution $p_{\mu}$ (Jaynes 2003); we have

$$
0=\delta \mathcal{L}_{\mathrm{TE}}\left(\left\{p_{\mu}\right\} ; \nu, \beta\right)=\sum_{\mu}\left[-\ln p_{\mu}-(\nu+1)-\beta E_{\mu}\right] \delta p_{\mu}
$$

for arbitrarily small variations $\delta p_{\mu}$ of $p_{\mu}$. Resolving (3) immediately gives

$$
p_{\mu}=\frac{\mathrm{e}^{-\beta E_{\mu}}}{Z(\beta)} \quad \text { with } \quad Z(\beta)=\sum_{\mu} \mathrm{e}^{-\beta E_{\mu}}
$$

as partition function (Jaynes 2003; Grandy 1987). Probability distribution (4) is Boltzmann probability (1) expressed in its canonical form (Jaynes 2003; Grandy 1987). If the maximum entropy formalism tells us how to treat total energy preservation, noticeably it does not tell us why we choose this constraint over another. Formally the preservation of the total energy is part of the initial hypothesis or available information (Jaynes 2003; Grandy 1987) that we have on systems in thermal equilibrium.

For self-organized urban street networks, our only available information is scale-freeness. However scale-freeness is not a preserved quantity but rather a property (Stanley 1971). But, at the same time, scale-freeness of a self-organized information network may result from a self-similarity inherited from its self-organized city (Kalapala et al 2006; Batty 2008). Self-similarity is a symmetry (Mandelbrot 1982), a transformation that lets an object or a system stay invariant. Symmetries play a fundamental role in modern physics (Gross 1996; Romero-Maltrana 2015; García-Pérez et al 2018). A general consensus in physics is that an invariance to a transformation underlies a preserved entity, and vice versa (Gross 1996; Romero-Maltrana 2015). Let us see how this idea applies here. For our purpose, we must first rewrite Lagrangian (2) in the more generic form

$$
\mathcal{L}_{f}\left(\left\{p_{\mu}\right\} ; \nu, \lambda\right)=-\sum_{\mu} p_{\mu} \ln p_{\mu}-v\left[\sum_{\mu} p_{\mu}-1\right]-\lambda\left[\sum_{\mu} p_{\mu} f\left(X_{\mu}\right)-\langle f(X)\rangle\right]
$$

where $X$ is an extensive quantity whose each value $X_{\mu}$ describes state $\mu$. An extensive quantity scales linearly under scaling transformations. The new second Lagrange multiplier $\lambda$ imposes our unknown constraint which expresses in terms of an unknown 
function $f$ acting on $X$. It literally coerces the mean value of $f(X)$ to have the constant value $\langle f(X)\rangle$. For the sake of demonstration, we will assume exact self-similarity. Accordingly, under the scaling transformation

$$
x \rightarrow \tilde{x}=\mathfrak{s} x,
$$

a self-similar (or homogeneous) function $\Phi(x)$ will transform as

$$
\Phi(x) \rightarrow \widetilde{\Phi(x)}=\Phi(\mathfrak{s} x)=\mathfrak{s}^{\alpha} \Phi(x)
$$

with $\alpha$ a scaling exponent (Stanley 1971, sec. 11.1). Here, the self-similarity invariance holds in the unknown probability distribution $p_{\mu}=p\left(X_{\mu}\right)$. Under transformation (6), $p_{\mu}$ remains unchanged as expected; we have

$$
p_{\mu} \rightarrow \widetilde{p_{\mu}}=\widetilde{p\left(X_{\mu}\right)}=\frac{p\left(\mathfrak{s} X_{\mu}\right)}{\sum_{\mu} p\left(\mathfrak{s} X_{\mu}\right)}=\frac{\mathfrak{s}^{\alpha} p\left(X_{\mu}\right)}{\sum_{\mu} \mathfrak{s}^{\alpha} p\left(X_{\mu}\right)}=p\left(X_{\mu}\right)=p_{\mu} .
$$

If we demand that Lagrangian (5) stays invariant under transformation (6), then

$$
\begin{aligned}
0 & =\left(\mathcal{L}_{f}\left(\left\{p_{\mu}\right\} ; v, \lambda\right)\right)^{\sim}-\mathcal{L}_{f}\left(\left\{p_{\mu}\right\} ; \nu, \lambda\right) \\
& =-\lambda\left\{\sum_{\mu} p_{\mu}\left[f\left(\mathfrak{s} X_{\mu}\right)-f\left(X_{\mu}\right)\right]-\langle f(\mathfrak{s} X)-f(X)\rangle\right\} \\
& =-\lambda\left\{\sum_{\mu} p_{\mu}\left[f\left(\mathfrak{s} X_{\mu}\right)-f(\mathfrak{s})-f\left(X_{\mu}\right)\right]-\langle f(\mathfrak{s} X)-f(\mathfrak{s})-f(X)\rangle\right\}
\end{aligned}
$$

for any scaling factor $\mathfrak{s}$ and any possible probability distribution $p_{\mu}$. Hence, the unknown function $f$ satisfies the functional equation

$$
f(\mathfrak{s} x)=f(\mathfrak{s})+f(x) .
$$

When $X$ takes only positive values $x$, the most general solution of (10) which is continuous is

$$
f(x)=K \ln x
$$

with $K$ a constant (Aczél 1966, Th. 2.1.2(2)). Substituting solution (11) into the generic Lagrangian (5) gives the self-similar Lagrangian

$$
\mathcal{L}_{\mathrm{SE}}\left(\left\{p_{\mu}\right\} ; v, \lambda\right)=-\sum_{\mu} p_{\mu} \ln p_{\mu}-v\left[\sum_{\mu} p_{\mu}-1\right]-\lambda\left[\sum_{\mu} p_{\mu} \ln X_{\mu}-\langle\ln X\rangle\right]
$$

once the useless constant $K$ is absorbed. One easily verifies that (12) remains indeed unchanged under the scaling transformation (6). The corresponding most plausible probability distribution $p_{\mu}$ yields the stationary equation

$$
0=\delta \mathcal{L}_{\mathrm{SE}}\left(\left\{p_{\mu}\right\} ; \nu, \lambda\right)=\sum_{\mu}\left[-\ln p_{\mu}-(v+1)-\lambda \ln X_{\mu}\right] \delta p_{\mu}
$$

whose solution readily writes 


$$
p_{\mu}=\frac{X_{\mu}^{-\lambda}}{Z(\lambda)} \quad \text { with } \quad Z(\lambda)=\sum_{\mu} X_{\mu}^{-\lambda}
$$

in the canonical form. One quickly checks that probability distribution (14) is invariant under the scaling transformation (6), as expected. This probability distribution is known as the discrete Pareto probability distribution (Clauset et al 2009). Let us summarize our result as follows. What the maximum entropy formalism (Jaynes 2003; Grandy 1987; Lawrence 2019) combined with the symmetry-conservation correspondence idea (Gross 1996; Romero-Maltrana 2015) tells us about statistically self-similar steady fluctuations is threefold:

(i) They follow a discrete Pareto probability distribution with the self-similar scaling exponent as scaling exponent.

(ii) They preserve on average the logarithm of an extensive quantity.

(iii) Their equilibrium parameter is the self-similar scaling exponent itself.

However, it can tell us nothing about the nature of the extensive quantity.

Thusly, the prerequisite to Monte Carlo methods for self-organized urban street networks can be expressed as follows. At scaling equilibrium, the probability $p_{\mu}$ for a selforganized urban street network to develop its streets in any state $\mu$ is assumed to yield the discrete Pareto distribution

$$
p_{\mu} \propto \mathrm{e}^{-\lambda \ln X_{\mu}}
$$

with $X_{\mu}$ the value at state $\mu$ of an extensive quantity $X$ and $\lambda$ the scaling exponent. Still, it remains to make a genuine hypothesis on the extensive quantity $X$.

\section{A surprisal-driven system}

In our context a state $\mu$ is a possible information network, namely a possible configuration of streets, that an urban street network can develop (see Fig. 1 for illustration). Previous investigations show that an information network of a self-organized urban street network typically underlies scale-freeness (Porta et al 2006; Crucitti et al 2006; Jiang et al 2008). Therefore, as shown in previous section, the distribution of their nodes (streets) preserves on average the logarithm of an extensive quantity, so that this distribution is most plausibly a discrete Pareto distribution of this extensive quantity. This extensive quantity cannot be specified due to our lack of knowledge on information networks of self-organized urban street networks.

However, the simplest assumption we can make is that a self-organized urban street network is a self-similar mesoscopic system whose mesoscopic objects have equiprobable configurations. Namely, we apply to our mesoscopic objects the principle of indifference (Jaynes 2003; Lawrence 2019). We may call such a system a self-similar Boltzmann-mesoscopic system. Our extensive quantity becomes then the number of equiprobable configurations of the mesoscopic objects. Let us denote by $\operatorname{Pr}(\Omega)$ the probability for a mesoscopic object to have $\Omega$ possible equiprobable configurations, and by $o(\Omega)$ a mesoscopic object having effectively $\Omega$ possible equiprobable configurations. With these notations, we may say that each mesoscopic object $o(\Omega)$ has $\Omega$ as extensive quantity. Thence, for each mesoscopic object $o(\Omega)$, our extensive quantity logarithm $\ln \Omega$ 
interprets itself either as the Boltzmann entropy of $o(\Omega)$ or as the surprisal associated to each configuration of $o(\Omega)$. Surprisal (or surprise, or information content) $\mathrm{Su}=-\ln \circ \mathrm{Pr}$ measures uncertainty, astonishment, and knowledge attached to an event (Tribus 1961; MacKay 2003; Applebaum 2008; Stone 2015; Lawrence 2019). While the average of surprisal over all the possible events gives their (Shannon) entropy, the surprisal attached to a possible event pertains its cognitive magnitude. When an event expected to be rare occurs, we are surprised and we feel that we learn a lot: the larger the uncertainty before the event, the greater the astonishment at the event, the wider the knowledge after the event (MacKay 2003; Applebaum 2008; Lawrence 2019). And vice versa. So that, compared to the entropy interpretation, the surprisal interpretation appears in essence finer and more cognitive. For these reasons, we may favour the surprisal interpretation. The preserved moment $\sum_{\Omega} \operatorname{Pr}(\Omega) \ln \Omega$ interprets then itself as an amount of surprisal that equilibria preserve on average. We interpret thusly steady fluctuations as a manifestation of uncertainties, astonishments, and knowledges whose the magnitudes remain on average the same. Presuming that this manifestation actually reflects a social process, each equilibrium becomes then a match between steady fluctuating configurations of streets and how city-dwellers comprehend their own urban street network (Dover 2004; Benoit and Jabari 2019a, b). We may expect that their comprehension reflects their agility and proficiency to navigate their own urban street network in normal or disrupted traffic.

With this assumption, the probability $p_{\mu}$ for a self-organized urban street network to develop an information network (or a configuration of streets) $\mu$ yields

$$
p_{\mu} \propto \prod_{o_{\mu} \in\left\{S_{\mu}, j_{\mu}\right\}} \Omega_{o_{\mu}}^{-\lambda}=\mathrm{e}^{-\lambda S_{\mu}}
$$

with

$$
S_{\mu}=\sum_{o_{\mu} \in\left\{s_{\mu}, j_{\mu}\right\}} \ln \Omega_{o_{\mu}}
$$

the total amount of surprisal for information network $\mu$; the product (the sum) is over the streets $s_{\mu}$ and junctions $j_{\mu}$ of information network $\mu$. Along the interpretation developed in the previous paragraph, the total amount of surprisal $S_{\mu}(17)$ quantifies the comprehension of the city-dwellers for information network $\mu$. Thus, accordingly, it is their comprehension that drives probability distribution (16), that is, the statistical equilibrium of their own urban street network.

\section{The Metropolis acceptance ratio}

Now that we have set up the fluctuating statistical model of our system, we are ready to implement the emblematic part of the Metropolis algorithm. The Metropolis algorithm holds its specificity among Monte Carlo methods in the implementation details of the condition of detailed balance (Newman and Barkema 1999; Landau and Binder 2015). This condition assures both that (i) each Markov chain (or sequence) reaches an equilibrium and that (ii) the equilibrium states follow the prescribed probability distribution. It applies, technically, to the probability $P(\mu \rightarrow v)$ of generating a state $v$ from a given state $\mu$ which is called the transition probability; along the constraint 


$$
\sum_{v} P(\mu \rightarrow v)=1
$$

the transition probabilities $P(\mu \rightarrow v)$ must satisfy the detailed balance equation

$$
p_{\mu} P(\mu \rightarrow v)=p_{\nu} P(\nu \rightarrow \mu) .
$$

Each transition probability $P(\mu \rightarrow \nu)$ may be split into two parts as

$$
P(\mu \rightarrow v)=g(\mu \rightarrow v) A(\mu \rightarrow v) .
$$

The selection probability $g(\mu \rightarrow v)$ is a probability imposed to our algorithm for generating a new state $v$ given a state $\mu$, while the acceptance ratio $A(\mu \rightarrow v)$ gives the odds of accepting or rejecting the move to state $v$ from state $\mu$. For the Metropolis algorithm, the selection probabilities $g(\mu \rightarrow v)$ for all permitted transitions are equal. Scheme (20) along this choice reduces the detailed balance equation (19) into a ratio equation for the acceptance ratios $A(\mu \rightarrow v)$; we have

$$
\frac{P(\mu \rightarrow v)}{P(\nu \rightarrow \mu)}=\frac{g(\mu \rightarrow v) A(\mu \rightarrow v)}{g(v \rightarrow \mu) A(v \rightarrow \mu)}=\frac{A(\mu \rightarrow v)}{A(v \rightarrow \mu)}=\frac{p_{v}}{p_{\mu}} .
$$

The last equality tells us that the odds of accepting or rejecting a move between two states are in favour to the more likely of them. This is common sense. Nonetheless, this still leaves open numerous possibilities. For the Metropolis algorithm, the more likely moves are assumed certain, while the less likely moves get their odds adjusted with respect to (21); we read

$$
A(\mu \rightarrow v)= \begin{cases}p_{\nu} p_{\mu}^{-1} & \text { if } p_{\nu}<p_{\mu} \\ 1 & \text { otherwise }\end{cases}
$$

For our statistical model (16), the Metropolis acceptance ratio $A(\mu \rightarrow v)$ takes the more familiar form

$$
A(\mu \rightarrow v)= \begin{cases}\mathrm{e}^{-\lambda\left(S_{v}-S_{\mu}\right)} & \text { if } S_{v}-S_{\mu}>0 \\ 1 & \text { otherwise }\end{cases}
$$

That is to say, if the newly selected information network $v$ has a total amount of surprisal $S_{\nu}$ strictly greater than the current one $S_{\mu}$, we accept to replace the current information network $\mu$ by the newly selected one $v$ with the probability given above; otherwise, we accept with certainty.

\section{Two simple ergodic single-junction dynamics}

The state-of-the-art generating paradigms are not dynamics. This is primarily because they build each information network from scratch. To be a dynamics, they should instead create a new information network from the current one. An analysis of their street-oriented paradigm gives us clues to design relevant ergodic dynamics. This second achievement of our paper permits us to concretely adapt the Metropolis algorithm to self-organized urban street networks. 


\section{A street is an exclusive joined sequence of street-segments}

For information networks, nodes are streets, basically an exclusive sequence of successive street-segments that are joined at junctions according to some paradigms. By exclusive we mean that a street-segment can only belong to a single street. This is the perspective used in the state-of-the-art literature (Jiang and Claramunt 2004; Rosvall et al 2005; Porta et al 2006; Jiang et al 2008; Masucci et al 2014).

An immediate paradigm is the "named street" paradigm (Jiang and Claramunt 2004; Jiang et al 2008) which simply reproduces cadasters ${ }^{2}$. Since for some cities a cadaster may not exist, or simply reflect local habits and customs, some studies have considered generic substitutes instead. The choice of the paradigm may then ponder social and geographical phenomena. A relevant parameter has appeared to be the deflection angle between two adjacent street-segments (Jiang et al 2008; Molinero et al 2017). Figure 2 illustrates the notion of deflection angle in our context through two typical junctions. If beyond some threshold angle any joining has to be excluded, many possibilities remain open.

Three paradigms based on deflection angles have been mainly used to generate information networks. Basically these paradigms are nonoverlapping walks governed by a join principle. The every-best-fit join principle (Porta et al 2006; Jiang et al 2008) acts at every junction by joining its street-segment pairs in increasing order of their deflection angles, until applicable. The self-best-fit join principle (Viana et al 2013; Jiang et al 2008) and self[-random]-fit join principle (Jiang et al 2008) act sequentially on growing streets, until applicable, by randomly seeding them with a not-yet-selected street-segment before recursively appending, until applicable, one of the not-yet-appended street-segments. The self join principles differ only in the choice of the not-yet-selected street-segment to append. Figure 3 illustrates how the inner recursion can construct an entire street; supplementary Animation A1 (Additional file 1) shows how the full machinery can achieve a complete configuration of streets. The self-best-fit join principle selects the one forming the smallest deflection angle; the self[-random]-fit join principle selects at random. By construction, these three joint principles fall into two categories. The every-best-fit join principle is local and almost deterministic ${ }^{3}$; the two self join principles are global and random. The latters clearly differ nevertheless in the degree of their randomness. Unsurprisingly, due to their walk-oriented construction, the two self join principles have appeared, against well-founded cadasters and transportation traffic in terms of correlation, more realistic (Jiang et al 2008). They thusly show that the deflection angle is a suitable parameter for generating information networks. However, the same walk-oriented construction renders them not easily tractable. In short, even though it provides a suitable parameter, the state-of-the-art approach can not be used to build an easily tractable dynamics.

\footnotetext{
${ }^{2}$ A cadaster is a comprehensive land register maintained by either local or central authorities. Cadasters have been used, in some parts of the world, for levying taxes, raising armies, setting ownerships, etc.

3 The every-best-fit join principle is almost deterministic in the sense that it resolves at random the very rare occurrences of equality between deflection angles.
} 


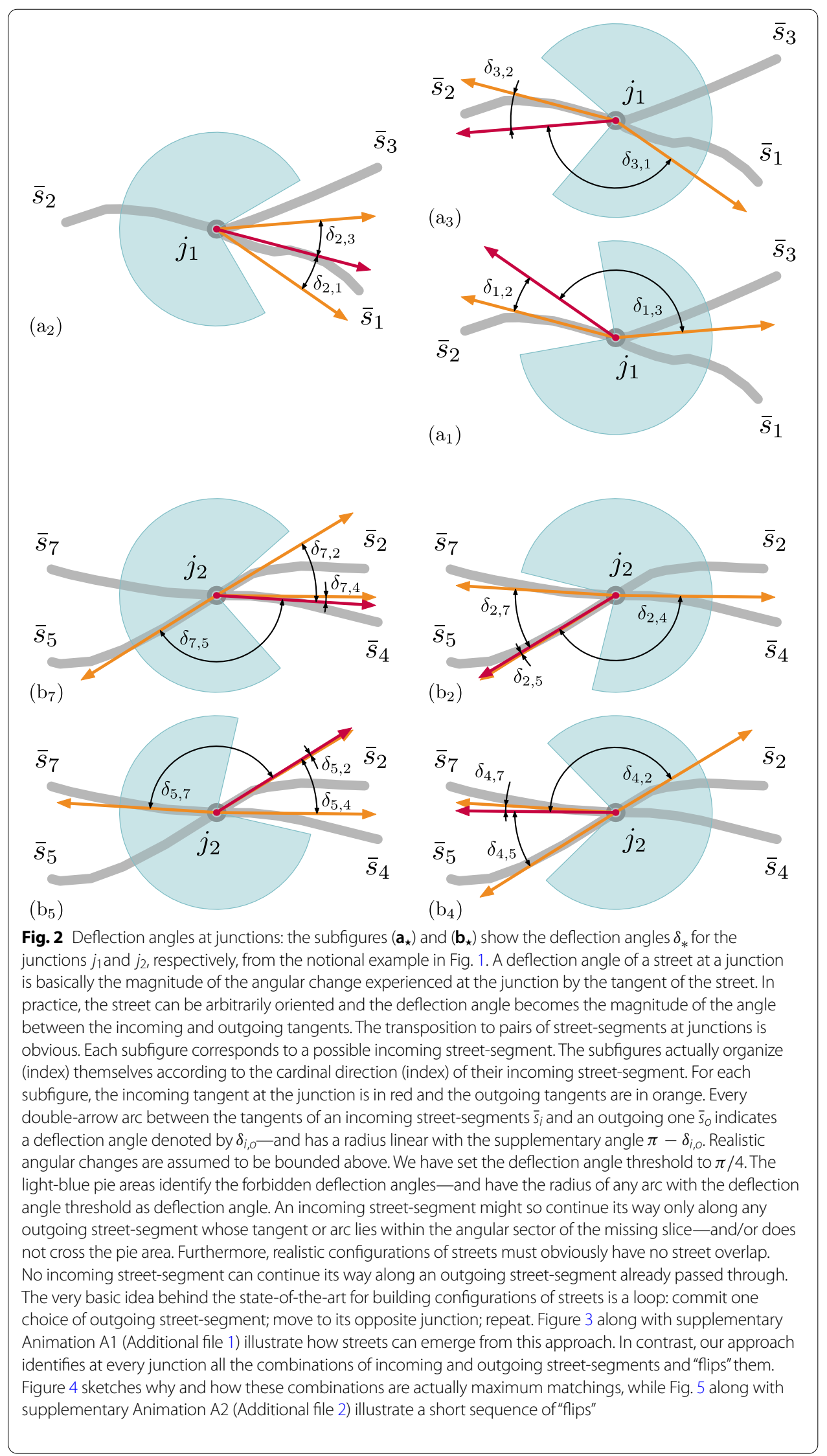


(See figure on next page.)

Fig. 3 State-of-the-art construction paradigm for configurations of streets: the frames $\left(\mathbf{s}_{\star}^{*}\right)$ show this paradigm steps for constructing street $r_{c}$ on street map $(\mathbf{m})$ from the notional example in Fig. 1. Our illustration assumes that streets $r_{a}$ and $r_{b}$ were constructed previously and that the remaining streets will be constructed afterward. The superscript and subscript of each frame label indicate the street under construction and the step order, respectively. Each street-segment in colour already belongs to a street: when the colour is vivid and the line is solid, the street was committed; when the colour is pallid or the line is vividly dashed, the street is under construction. Each street-segment in grey is a candidate for belonging to a new street. We attribute to each street a particular colour. The construction goes like this. Initial stage $\left(s_{0}^{c}\right)$ : no street-segment is yet assigned to street $r_{c}$. Seeding step $\left(s_{1}^{c}\right)$ : pick at random one candidate street-segmentthe seed street-segment is in pallid orange and marked with an orange bold square. Orientation step $\left(s_{2}^{c}\right)$ : orient at random the seed street-segment and move toward the head junction - the square mark is now a pentagonal "home plate" indicating the orientation, the path moved along from the mark to the head junction $j_{1}$ is now vividly dashed, the excluding pie aligned with the incoming tangent at $j_{1}$ immediately identifies along which outgoing street-segments the street might continue (see Fig. 2). Appending loop steps $\left(s_{3}^{c}\right)-\left(s_{5}^{c}\right)$ : arbitrarily continue at the head junction along any valid outgoing street-segment (see Fig. 2) while applicable - at $j_{1}$ the street might continue toward either $i_{1}$ or $j_{3}$ (see Fig. 2a 2 ), the latter choice was arbitrarily taken; at $j_{3}$ the street might continue toward either $i_{3}$ or $j_{5}$, as the former choice was no more possible only the latter could be taken; at $j_{5}$ the street can only continue toward $i_{7}$; at $i_{7}$ the street can no more continue so that the recursion ended. Inverting step $\left(s_{6}^{c}\right)$ : move toward the tail junction and formally invert orientationthe forward recursion lets now place to a backward recursion, the pentagonal mark has flipped its orientation and has rounded its tail to mark the epoch. Prepending loop steps $\left(s_{7}^{c}\right)-\left(s_{8}^{c}\right)$ : arbitrarily continue at the tail (formal head) junction along any valid outgoing street-segment (see Fig. 2) while applicable-at $j_{2}$ the street might continue toward either $j_{4}$ or $j_{6}$ (see Fig. $2 b_{2}$ ), the former choice was arbitrarily taken; at $j_{4}$ the street can only continue toward $i_{6} ;$ at $i_{6}$ the street can no more continue so that the backward recursion ended. Commit step $\left(s_{9}^{c}\right)$ : commit the new achieved street and loop forward to build the next street until applicable - the now achieved street $r_{c}$ is in solid line, its mark is an unbold circle, and it has a label; retrospectively, this step leads to Initial stage $\left(s_{0}^{d}\right)$ for the next street $r_{d}$ while the above Initial stage $\left(s_{0}^{c}\right)$ appears to result from Commit step $\left(s_{6}^{b}\right)$ for the previously committed street $r_{b}$; the construction of streets loops until no more street-segment is unassigned. The arbitrary choices in Appending and Prepending loop steps are actually join principles (see "A street is an exclusive joined sequence of street-segments" section). Supplementary Animation A1 (Additional file 1) shows a complete construction of the configuration of streets on street map (m)

\section{A junction is a matching of street-segments}

For information networks, edges are junctions, essentially an exclusive set of singletons and pairs of street-segments that are isolated or paired according to the ongoing streets. By exclusive we mean that a street-segment can only belong either to one singleton or to one pair. Such a set is, in graph theory, a matching (Pemmaraju and Skiena 2003). To the best of our knowledge, this is the first work that mentions this perspective.

The graph theory perspective can apply on junctions as follows. First, inspired by the dual network representation of urban street networks, we may represent every streetsegment attached to a junction by a node. Let us put each node at the intersection of its associated street-segment with a circle centred at the junction. Second, we may link pair of nodes whose associated street-segments have a deflection angle smaller than the deflection angle threshold. Figure 4 illustrates in its two first rows these two steps for three realistic junctions. The resulting graph clearly depends on the deflection angle threshold: when it is set to the flat angle $\pi$, the graph is a complete graph; when it is set to the zero angle 0 , the graph is an empty graph; otherwise the graph is an incomplete graph. We will call such a graph a junction graph. In general, a junction graph has no direct application for our purpose in the sense that any bunch of edges that share a common node (or adjacent edges) corresponds to a set of overlapping streets. In practice, we want a graph without any adjacent edge so that the graph corresponds to a set of nonoverlapping streets. Such a graph is, for a given junction, a matching subgraph (or 


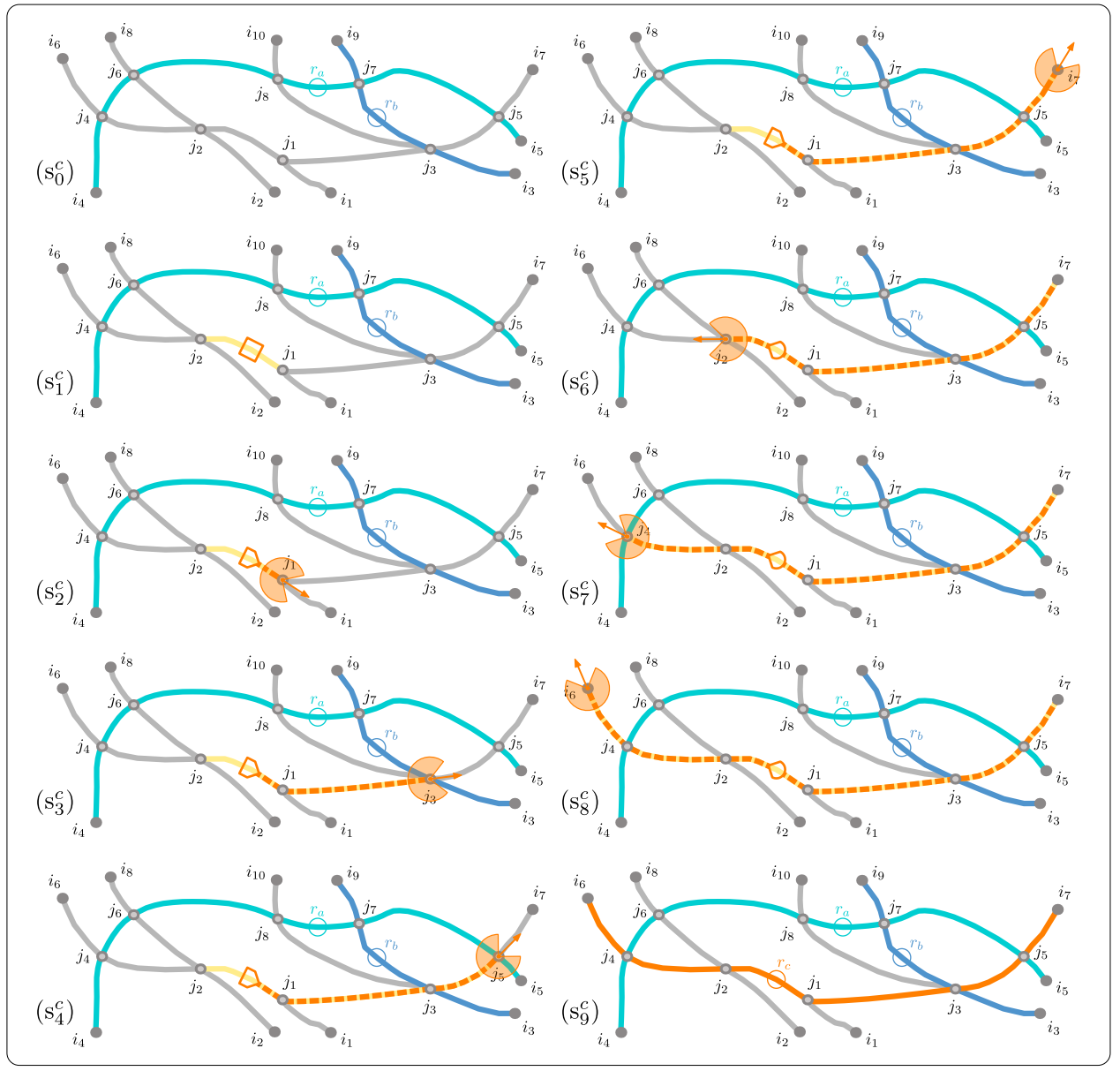

matching for short) (Pemmaraju and Skiena 2003) of its a junction graph. In short, we are interested by the set of matchings of the junction graphs. The number of matchings of a graph is called the Hosoya index (Hosoya 1971). We will denote the Hosoya index of the junction graph of a junction $j$ by $\mathscr{Z}_{j}$. Also notice that a matching can be saturated in the sense that it cannot be expanded to another matching by adding any edge of the underlying graph. Such a matching is called a maximal matching (Pemmaraju and Skiena 2003). Figure 4 gives in its fourth row the set of maximum matchings we can derive for each of its junctions. We will denote the number of maximal matchings of the junction graph of a junction $j$ by $\mathscr{Y}_{j}$; we have

$$
1 \leqslant \mathscr{Y}_{j} \leqslant \mathscr{Z}_{j}
$$

Let us now describe the previous generating paradigms in terms of matchings. The "named street" paradigm selects the matching as implicitly recorded in cadasters. The every-best-fit join principle chooses for each junction the maximal matching which is optimal in terms of deflection angle distribution. The two self join principles operate at every junction on the set of matchings by successive visits. This becomes more apparent when we interpret their concrete implementations as nonoverlapping walks that haphazardly visit every junction several times. Each visit either steps forward or terminates the 


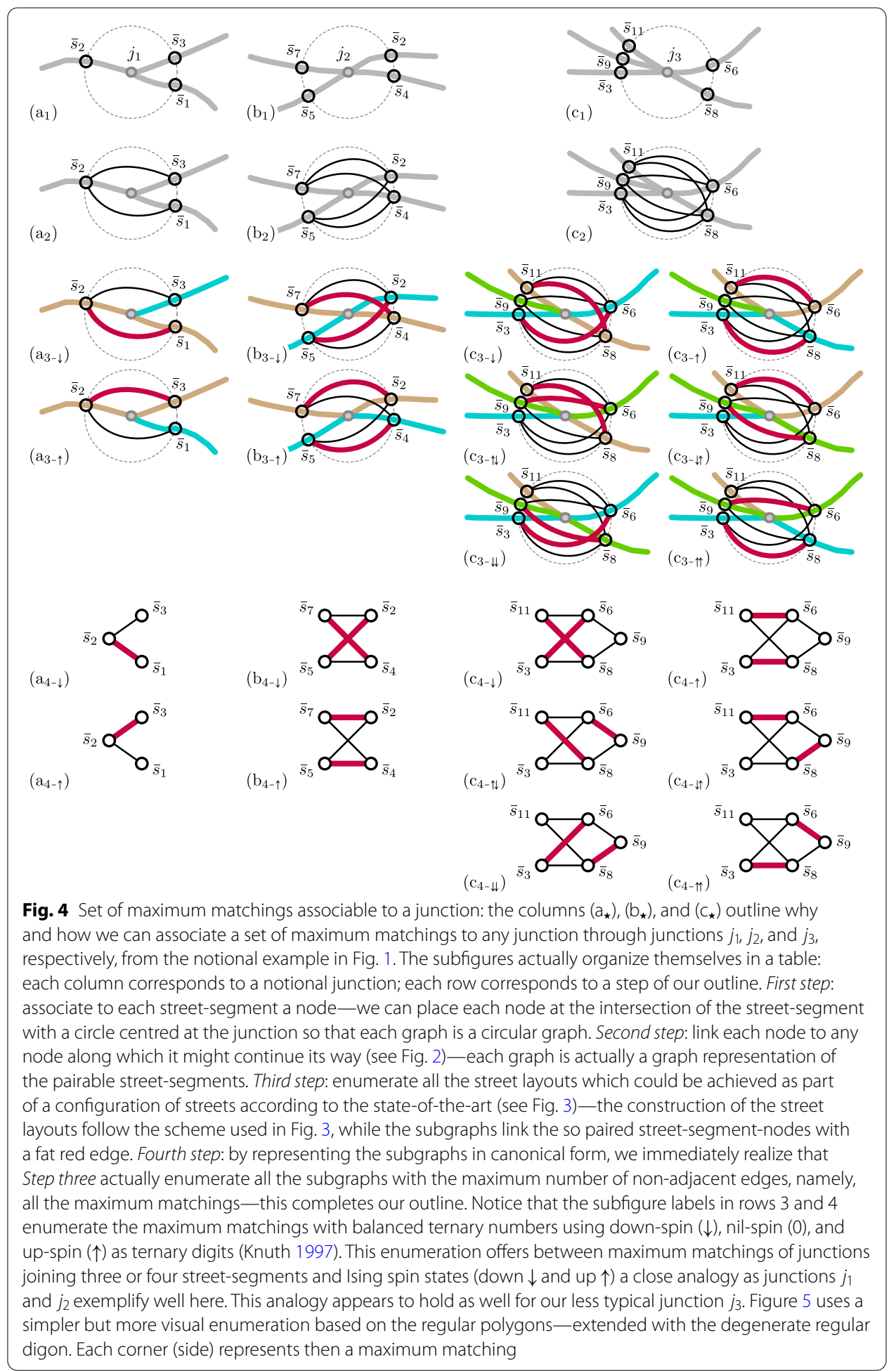

walk, that is, each visit selects a subset of matchings. This selection process reveals itself in supplementary Animation A1 (Additional file 1). For the self-best-fit join principle, the move is optimal in terms of deflection angle; for the self[-random]-fit join principle, 
the move is random. Over the visits the subset of matchings decreases until it contains only one matching. This remaining matching is a maximal matching since every walk terminates only when no more street-segment is attachable. Figure 4 draws in its third row the end results of these repeated visits along their maximum matching for each of its junctions. Actually, Fig. 4 sketches, through three realistic junctions, why and how to any junction corresponds a set of maximum matching. The so isolated maximal matchings give the generated information network. In other words, the self join paradigms interpret themselves now as an intricate haphazard fashion to pick for every junction a maximal matching. Thusly, the matching viewpoint allows us to slightly disentangle the two most pertinent join paradigms.

\section{The single-junction-switch and -flip dynamics}

The previous slightly untangled description actually leads to a disembodied form of the self join paradigms with all their underlying principles removed. This is exactly what an ergodic dynamics is about. To the best of our knowledge, no ergodic dynamics has been reported so far for generating configurations of streets.

To begin with, let us deliberately ignore for a while the nonoverlapping walk machineries. The self join paradigms reduce then to choose for every junction a maximal matching regardless of the matchings of the other junctions. So the elementary disembodied dynamics that occurs at junctions is to set up in an independent way a maximal matching. The new set up will generally change the maximal matching into another maximal one. For clarity, this dynamics does not alter the urban street network but rather transforms the information network into another, since the new maximal matching sets a new layout for some of the streets that cross the junction. By now we are able to tell that this dynamics is ergodic. An ergodic dynamics is a dynamics which from any state can reach any other state after a finite number of iterations. It is indeed obvious that we can get from any information network to any other by changing one by one each of the maximal matchings by which the two information networks differ. We coined this dynamics, following the literature (Newman and Barkema 1999) and as an obvious analogy to railroad switches, the single-junction-switch dynamics. The restriction to consider only maximal matchings is inherited from the join principles. This restriction is arbitrary in the sense that it is not actually imposed by physical constraints. In fact, the reasoning held above for the single-junction-switch dynamics evidently holds for any arbitrary choice of subset of matchings. For completeness, we coined the dynamics that involves all matchings the single-junction-flip dynamics. Let us recap along these lines. Assuming an entire urban street network, the single-junction-switch dynamics is an ergodic dynamics which switches the maximal matching of a single junction into another maximal one, while the single-junction-flip dynamics is an ergodic dynamics which flips the matching of a single junction into another.

Using the single-junction-switch or -flip dynamics ensures that our Metropolis algorithm fulfils the condition of ergodicity. It remains however to specify how we select from a given information network a new one which differs by only one dynamics step. For the sake of simplicity, we will only consider the single-junction-switch dynamics in the following. The choice of the Metropolis algorithm imposes (Newman and Barkema 1999; Landau and Binder 2015) that the selection probabilities $g(\mu \rightarrow v)$ for each 
possible new information network $v$ after one dynamics step are all chosen equal-the selection probabilities for all other information networks are set to zero. For an entire urban street network, one dynamics step enables to reach each of the $\mathscr{Y}_{j}-1$ new maximal matchings of every junction $j$. Hence the number of possible new information networks that we can reach after one dynamics step from a given information network is the total number of maximal matchings

$$
\mathscr{N}=\sum_{j} \mathscr{Y}_{j}
$$

minus the number of junction $N$. Therefore we count $\mathscr{N}-N$ non-zero selection probabilities $g(\mu \rightarrow v)$, and each of them takes the value

$$
g(\mu \rightarrow v)=\frac{1}{\mathscr{N}-N}
$$

In practice we can realize this selection in two easy steps. First we pick at random a junction $j$ with probability proportional to $\mathscr{Y}_{j}-1$. Then we choose at random a new maximal matching among the $\mathscr{Y}_{j}-1$ possible new maximal matchings of junction $j$.

\section{Another variant of the single-spin-flip Metropolis algorithm ?}

When all junctions have two maximal matchings, the single-junction-switch dynamics is formally equivalent to the single-spin-flip dynamics on the original Ising model. Our two above achievements actually combine to give another variation on the single-spinflip Metropolis algorithm theme. This algorithm is a computational interpretation of the Ising model. A brief comparison provides basic physical insights and a simple clue for a crossover as scaling varies.

\section{Informal implementation}

As summary of our above results, let us informally implement our adaptation of the Metropolis algorithm to urban street networks as follows.

First, we choose randomly a junction $j$ with probability proportional to its number of maximal matchings $\mathscr{Y}_{j}$ minus $1, \mathscr{Y}_{j}-1$; its street-segments will be laid out according to some maximal matching $\mathrm{M}_{j}$. Second, we pick at random a new maximal matching $\widetilde{\mathrm{M}}_{j}$ not identical to $\mathrm{M}_{j}$ among the remaining $\mathscr{Y}_{j}-1$ available possibilities. Third, we calculate the change in the total amount of surprisal $\Delta S$ that would result if we were to lay out this change to this junction. Ultimately, with acceptance probability

$$
A= \begin{cases}\mathrm{e}^{-\lambda \Delta S} & \text { if } \Delta S>0 \\ 1 & \text { otherwise }\end{cases}
$$

either accept or reject the change.

Properly speaking, this informal implementation encodes the single-junction-switch Metropolis algorithm for urban street networks. We let the reader to elaborate the 

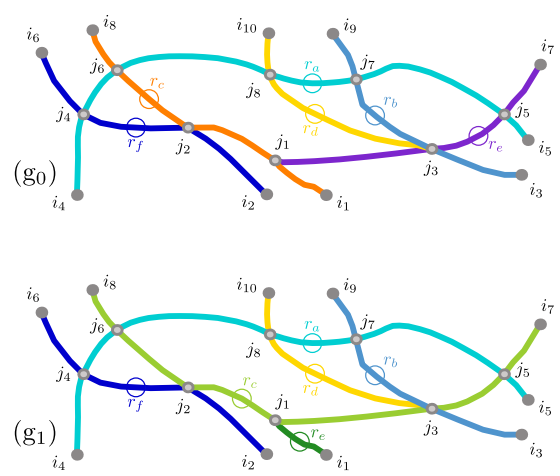

$\left(\mathrm{g}_{\frac{1}{2}}\right)$

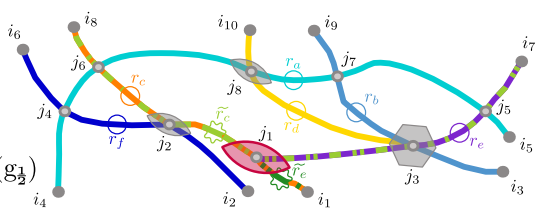

$\left(\mathrm{g}_{2}\right)$

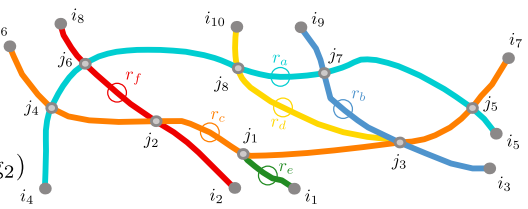

$\left(\mathrm{g}_{1 \frac{1}{2}}\right)$

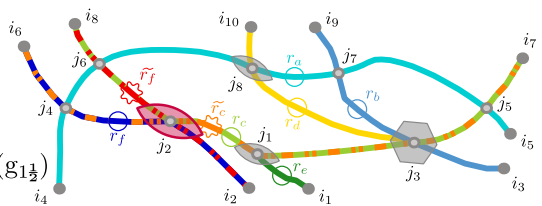

$\left(\mathrm{g}_{3}\right)$

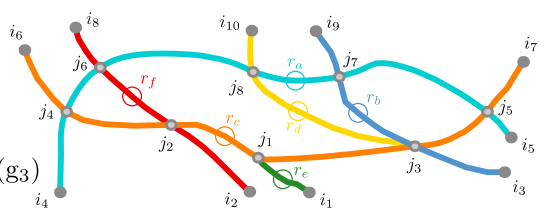

$\left(\mathrm{g}_{2 \frac{1}{2}}\right)$

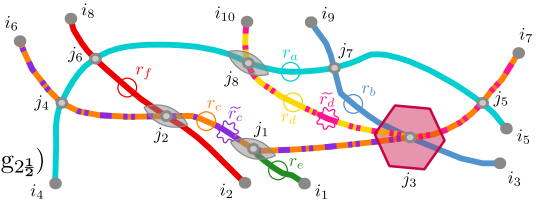

Fig. 5 Single-junction-switch Metropolis algorithm for configurations of streets: the frames $\left(g_{\star}\right)$ show how this algorithm may evolve from the configuration of streets on street map $\left(m^{\prime}\right)$ to the one on street map $(m)$ from the notional example in Fig. 1. The subscript of each frame label indicate the generation time. The figure reads from top to bottom. It actually separates generated configurations of streets from intermediate computational steps in two complementary ways: it shifts (resp. epochs) the formers to the left column (resp. with integers) and the latters to the right column (resp. with half-integers). The right frames display more information. In particular each switchable junction is marked with an extended regular polygon whose the corners (sides) enumerate its maximum matchings (see Fig. 4): when the polygon is in grey, the junction is resting; when the polygon is in red and notably bigger, the junction is switching. As for the state-of-the-art construction paradigm in Fig. 3, we attribute to each street a particular colour. In clear contrast, however, all streets are here fully constructed. The streets in dashed lines are not under construction but instead under challenge as follows. The dashing actually indicates coexistence of old streets with new ones. Each old street keeps its colour, its circle mark, and its label. Each new street emerges with a new color, a wavy-circle mark, and a tilded label. The new streets result from the new street layout at the switching junction. This change leads so to a new configuration of streets $v$ that competes with the old one $\mu$. This is the actual ongoing challenge. The Metropolis algorithm resolves such challenges by either accepting or rejecting change $\mu \rightarrow v$ with an acceptance ratio $A(\mu \rightarrow v)$ in an optimal way. After a sufficient number of generations, the configurations of streets reach a prescribed statistical equilibrium — provided the equilibrium is sustainable. Our prescribed statistical equilibrium follows from the assumption that self-organized urban street networks are statistically self-similar. It is a Boltzmann-like distribution with a total amount of surprisal (information) instead of energy and the scaling as equilibrium parameter (see formula (16)). Our odds of accepting or rejecting new configurations of streets favour the less surprising ones (see formulae (23) and (30)). The algorithm goes like this (see "Informal implementation" section). Note first that only the four junctions $j_{1}, j_{2}, j_{3}$, and $j_{8}$ are actually switchable: junction $j_{3}$ has $\mathscr{Y}_{3}=6$ maximum matchings (see Fig. $4 c$ ); junctions $j_{1} j_{2}$, and $j_{8}$ have $\mathscr{Y}_{1}=\mathscr{Y}_{2}=\mathscr{Y}_{8}=2$ maximum matchings (see Fig. $4 a$, b); the remaining junctions $j_{\circ}$ have obviously $\mathscr{Y}_{\circ}=1$ maximum matching. So each move will first choose randomly one junction among junctions $j_{1} j_{2}, j_{3}$, and $j_{8}$ with probabilities $\frac{1}{8}, \frac{1}{8}, \frac{5}{8}$ and $\frac{1}{8}$, respectively. Each move will second pick uniformly at random a new maximum matching. There will be $\mathscr{Y}_{3}-1=5$ choices for $j_{3}$ and $\mathscr{Y}_{1}-1=\mathscr{Y}_{2}-1=\mathscr{Y}_{8}-1=1$ choice for $j_{1} j_{2}$, and $j_{8}$. Each move will third calculate its change in amount of surprisal $\Delta S$ in view to compute its acceptance ratio $A$. Here the surprisal changes at mid-steps $\left(g_{1}\right),\left(g_{1} \frac{1}{2}\right)$, and $\left(g_{2 \frac{1}{2}}\right)$ are respectively $\Delta \widetilde{S}=\ln \frac{5}{9}, \Delta \widetilde{S}=0$, and $\Delta \widetilde{S}=\ln \frac{6}{5}$ (see"Working assumptions" section). Ultimately each move will either accept or reject the change with probability $A$. The layout changes at $\left(g_{1}\right)$ and $\left(g_{11}\right)$ are certain since they are less or equally surprising, the one at $\left(g_{21}\right)$ is accepted with probability $\exp \left(-\widetilde{\lambda} \ln \frac{6 \overline{5}}{5}\right)$ where $\widetilde{\lambda}$ is our effective equilibrium parameter (see formula (30)). Our illustration actually rejects the last move. Supplementary Animation A2 (Additional file 2) shows a longer sequence 
corresponding single-junction-flip Metropolis algorithm. Meanwhile, the reader may refer to Fig. 5 and supplementary Animation A2 (Additional file 2) for illustration.

\section{A brief comparison with Ising models}

The single-junction-switch (resp. single-junction-flip) Metropolis algorithm for our urban street network model mimics the classical single-spin-flip Metropolis algorithm for Ising models (Newman and Barkema 1999; Landau and Binder 2015; Berlinsky and Harris 2019; MacKay 2003). Nonetheless our model differs from them in three basic aspects:

(i) Our model is driven by scaling and surprisal (information) whereas Ising models are driven by temperature and energy. The parallel scaling-information versus temperature-energy $(\lambda, S) \leftrightarrow(\beta, E)$ pours into the discipline the all maturity of thermodynamics and statistical physics. This parallel is actually superseded by the maximum entropy formalism "in a disembodied form with all the physics removed" (Jaynes 2003). This formalism provides, for instance, numerical tools to compute for any information network measure (Newman 2018; Porta et al 2006) its linear response to arbitrary small scaling changes, namely its specific-heat-capacity-like coefficient or susceptibility (Newman and Barkema 1999; Grandy 1987; Jaynes 2003).

(ii) Junctions are nodes of a finite arbitrary planar graph while spins are classically attached to sites of an "infinite" regular lattice. Finiteness means that collective phenomena will get smoother. Arbitrariness renders our model closer to the Ising spin-glass models for which the values of the spin-spin interactions are no more constant but random (Newman and Barkema 1999; Landau and Binder 2015; MacKay 2003). Collective phenomena in Ising spin-glass models are more subtle and more intricate (Newman and Barkema 1999; Landau and Binder 2015).

(iii) The distribution of maximal matchings (resp. matchings) among junctions is heterogeneous while the distribution of spins among sites is classically homogeneous. That is, junctions have different numbers of maximal matchings (resp. numbers of matchings (Hosoya indices)) while spins have classically the same number of states or the same dimension. Because the number of street-segments attached to a junction is mostly three or four, the distribution of matching is expected to be statistically homogeneous with a bell-like distribution. This contributes to make our model even closer to the Ising spin-glass models.

On the other hand, it is noteworthy that the Ising models have became a toy model to crack phase transition and crossover phenomena (Berlinsky and Harris 2019; MacKay 2003). This raises the obvious question whether our model may actually undergo a crossover as scaling varies:

(iv) Our model experiences, as scaling increases, an ultra-small- to small-world crossover around the scaling value of 3 . The small-world effect is in effect a statement on geodesic (or shortest) distances between node pairs (Newman 2018). Their mean $\ell$ behaves in small-world networks as the logarithm of the number of nodes $N$, $\ell \sim \ln N$ (Newman 2018). The small-world effect becomes extreme in scale-free networks as the scaling $\lambda$ get smaller than 3 (Cohen and Havlin 2003): the mean 
geodesic distance $\ell$ behaves as $\ell \sim \ln \ln N$ when $2<\lambda<3$, as $\ell \sim \ln N / \ln \ln N$ at $\lambda=3$, and as $\ell \sim \ln N$ for $3<\lambda$. Thusly, since our generated information networks are scale-free, our model effectively undergoes a small-world crossover as scaling varies. Clearly its manifestation relies on the behaviour of the number of nodes $N$. A more substantial analytical work is however beyond the scope of the present paper. Meanwhile, notice that a geodesic distance counts in our context how many changes of street are required for a particular journey. That is, the mean geodesic distance reflects how rapidly on average city-dwellers can travel. Accordingly, the crossover diagram of the mean geodesic distance interprets itself as an efficiency diagram. This means, for instance, that our approach provides a method to analyze the relative efficiency of an actual configuration of streets.

\section{Equilibrium Metropolis simulations}

What we have achieved in the previous section is adapting to unplanned or self-organized urban street networks the Metropolis algorithm. Now, in this section, we exercise this adaptation in a case study. As case study, we select the urban street network of Central London (United Kingdom), which is a classical example of self-organized urban street network (Jacobs 1993).

\section{Working assumptions}

For the sake of illustration, we have made two suppositions. First, we have assumed that streets predominate junctions. Second, we have described the mesoscopic streets as asymptotic agent systems driven by social interactions (Dover 2004; Benoit and Jabari 2019a, b). According to this agent model, a number of vital connections $v$ dominates among the possible numbers of connection between agents. So that, the number of configurations $\Omega_{s_{\mu}}$ of street $s_{\mu}$ in configuration of streets $\mu$ becomes proportional to a power of its number of junctions $n_{s_{\mu}}$ (Benoit and Jabari 2019a, b); we have

$$
\Omega_{s_{\mu}} \propto n_{s_{\mu}}^{2 v}
$$

So the total amount of surprisal $S_{\mu}(17)$ in configuration of streets $\mu$ becomes

$$
S_{\mu}=2 v \sum_{s_{\mu}} \ln n_{s_{\mu}}
$$

up to an irrelevant constant; the sum is over the streets $s_{\mu}$ of configuration of streets $\mu$. Thusly our working assumptions bring out an effective scaling exponent $\widetilde{\lambda}$ along an effective total amount of surprisal $\widetilde{S}_{\mu}$; we read

$$
\widetilde{\lambda}=2 \lambda v \quad \text { and } \quad \widetilde{S}_{\mu}=\sum_{s_{\mu}} \ln n_{s_{\mu}} .
$$

The corresponding effective Metropolis acceptance ratio is literally the tilde version of formula (23); we get 

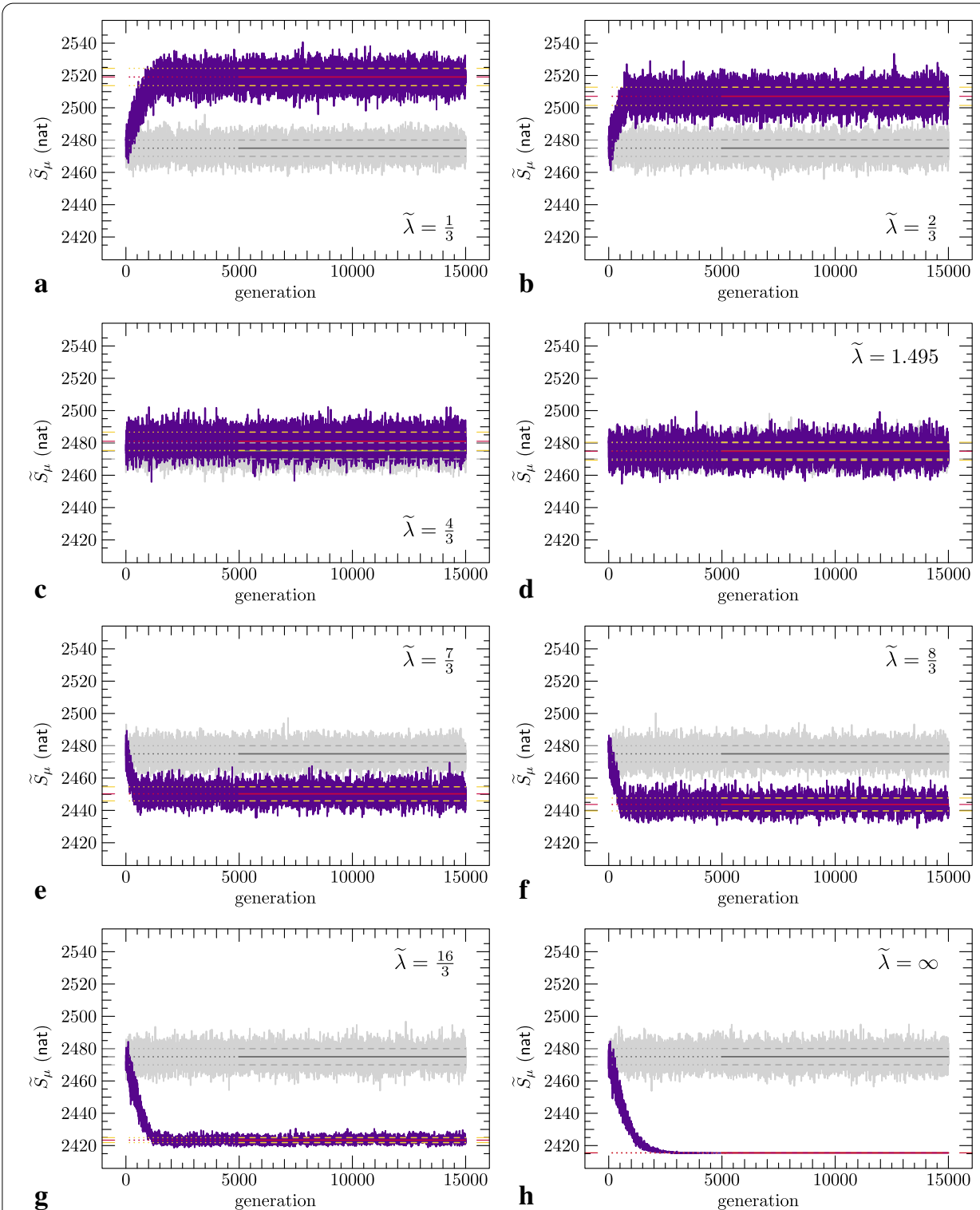

Fig. 6 Typical single-junction-switch Metropolis generation series for Central London (United Kingdom): the foreground purple generation series plot, for different effective scaling exponents $\widetilde{\lambda}=2 \lambda v$, typical simulations starting from a self-fit output; the background light-grey generation series plot, according to the same modus operandi, typical sequences of self-fit outputs. Each horizontal solid line drawn along a generation series plot represents its in-equilibrium mean value, the accompanying horizontal dashed lines indicate the associated standard-deviation bounds. Coloured lines, Metropolis generation series; greyed lines, sequences of self-fit outputs. The equilibria are assumed reached after the 5000 th generation. The following annealing parameters were used to algebraically cool down to the desired $\widetilde{\lambda}: \widetilde{\lambda}_{0}=1.495, \epsilon=10^{-3}$ and $m=500$. ( The starting cooling value $\lambda_{0}$ was chosen by hand so that the associated Metropolis equilibrium approaches on average self-fit outputs - as illustrated in (d); the special case $\widetilde{\lambda}=\infty$ in (h) corresponds to a full 'simulated annealing' process (Press et al 2007; Galassi et al 2009). )

$$
A(\mu \rightarrow v)= \begin{cases}\mathrm{e}^{-\tilde{\lambda}\left(\widetilde{S}_{\nu}-\widetilde{S}_{\mu}\right)} & \text { if } \widetilde{S}_{\nu}-\widetilde{S}_{\mu}>0 \\ 1 & \text { otherwise. }\end{cases}
$$



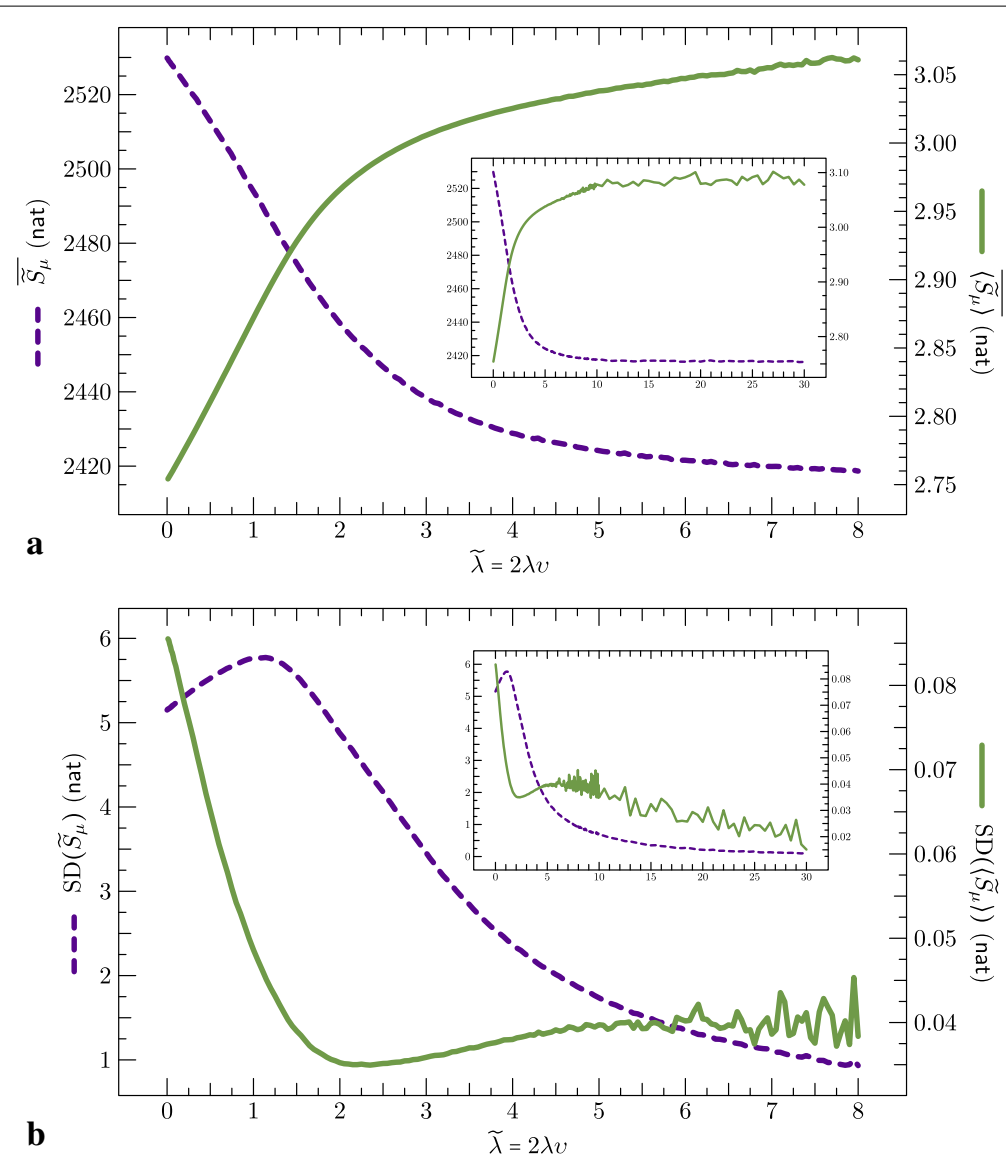

Fig. 7 Effective total and average amounts of surprisal versus effective scaling exponent for Central London (United Kingdom): green solid lines plot the mean (top) and standard-deviation (bottom) of the effective average amount of surprisal $\left\langle\widetilde{S}_{\mu}\right\rangle$; purple dashed lines plot the mean (top) and standard-deviation (bottom) of the effective total amount of surprisal $\widetilde{S}_{\mu}$ t the insets show their asymptotic behaviours. The annealing parameters are the same as in Fig. 6; the equilibria were assumed reached after the 5000 th generation as in Fig. 6; the mean and standard-deviation values were computed over 250000 in-equilibrium generations and averaged over 10 simulations. (We attribute the noise that waves the asymptotic branches to the poor quality of our map data (see Footnote 4))

Figure 5 along with supplementary Animation A2 (Additional file 2) show how our Metropolis adaptation can actually generate a sequence of configurations of streets.

\section{Equilibria}

\section{Single-junction-switch Metropolis generation series versus self-fit outputs}

Central London offers, as shown in Fig. 6, single-junction-switch Metropolis generation series that come to equilibria. The equilibria were attained from self-fit outputs through a basic algebraic annealing schedule (Newman and Barkema 1999; Press et al 2007; Galassi et al 2009). To paraphrase: increase (resp. decrease) the control effective scaling exponent $\widetilde{\lambda}_{c}$ to $\widetilde{\lambda}_{c}(1+\epsilon)\left(\right.$ resp. $\left.\tilde{\lambda}_{c} /(1+\epsilon)\right)$ after every $m$ accepted/rejected single-junction-switch moves up (resp. down) to the desired equilibrium effective scal-

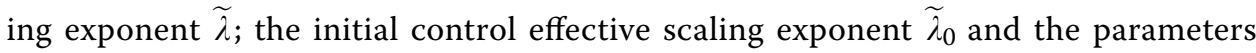
$\epsilon$ and $m$ are determined by experiment. This annealing schedule allowed us to reach 
equilibria for a range of effective scaling exponent $\tilde{\lambda}$ values large enough to capture the features of our system as follows.

The single-junction-switch Metropolis generation series exhibited in Fig. 6 show at least four noticeable properties:

(i) The sustained Metropolis equilibria $(a-f)$ are clearly comparable to the self-fit outputs in terms of order of magnitude of their means and fluctuations. This property holds, as shown Fig. 7, within a window grossly comprised between 1 and 5. We must always bear in mind that scaling exponents of real-world networks are typically comprised between 2 and 3 (Newman 2018).

(ii) The ground state, namely the sustained Metropolis equilibrium (h) attained for $\tilde{\lambda}=\infty$, lays below the self-fit outputs by about eleven times their standard-deviation. The ground state was obtained through a simulated annealing' (Press et al 2007; Galassi et al 2009).

(iii) The sustained Metropolis equilibrium (a) shows that there also exist equilibria that detach significantly from the self-fit outputs from above.

(iv) The sustained Metropolis equilibrium (d) shows that the single-junction-switch Metropolis algorithm can mimic quite well sequences of self-fit outputs.

These four properties lead us to claim that the single-junction-switch Metropolis algorithm generates series that are consistent with the self-fit outputs.

The effective total and average amounts of surprisal, $\widetilde{S}_{\mu}$ and $\left\langle\widetilde{S}_{\mu}\right\rangle$ respectively, exhibit in Fig. 7 at least two promising properties:

(i) Their means and their standard-deviations vary smoothly as a function of the effective scaling exponent $\tilde{\lambda}$ at least for values smaller than 4.5. For greater effective scaling exponent $\tilde{\lambda}$ values, our simulations get subject to noise: the effective total amounts of surprisal $\widetilde{S}_{\mu}$ and its standard-deviation $\operatorname{SD}\left(\widetilde{S}_{\mu}\right)$ continue to vary smoothly while they tend asymptotically to a constant; however, their average counterparts $\left\langle\widetilde{S}_{\mu}\right\rangle$ and SD $\left(\left\langle\widetilde{S}_{\mu}\right\rangle\right)$ experience noisy variations. ${ }^{4}$

(ii) They all experience a noticeable change of behaviour within the window comprised between 1 and 4 . The means of $\widetilde{S}_{\mu}$ and $\left\langle\widetilde{S}_{\mu}\right\rangle$ experience both a change of rate that leads them to their respective asymptotic plateau. The standard-deviations $\operatorname{SD}\left(\widetilde{S}_{\mu}\right)$ reach a maximum at right of 1 before decreasing towards an asymptotic plateau; the standard-deviations SD $\left(\left\langle\widetilde{S}_{\mu}\right\rangle\right)$ has the left profile of a Mexican-hat-left shape of a biquadratic curve-with a minimum around 2 .

The latter property strongly suggests that the relevant physics of our system occurs within the window comprised between 1 and 4, while the former property means that Monte Carlo studies within this window are feasible.

\footnotetext{
${ }^{4}$ We attribute the noise to the poor quality of our map data in their small streets and in their simplification of the junctions. The variations of $\widetilde{S}_{\mu}$ and $\mathrm{SD}\left(\widetilde{S}_{\mu}\right)$ remains relatively smooth because the Metropolis acceptance ratio (30) tends to smooth $\widetilde{S}_{\mu}$ itself by rejecting the inappropriate states-among them there are the inappropriate states resulting from "corrupted" data. By contrast, the variation of $\left\langle\widetilde{S}_{\mu}\right\rangle$ and SD $\left(\left\langle\widetilde{S}_{\mu}\right\rangle\right)$ are not smoothed by the Metropolis algorithm in any manner. Furthermore, "corrupted" layout at junctions cannot be rejected because (i) our working assumptions do not take into account junctions in the computation of $\widetilde{S}_{\mu}$ and because (ii) the single-junction-switch dynamics cannot break them since it only allows maximal layouts-this becomes evident as soon as the single-junction-flip dynamics is used since then $\left\langle\widetilde{S}_{\mu}\right\rangle$ and SD $\left(\left\langle\widetilde{S}_{\mu}\right\rangle\right)$ vary almost smoothly along their respective asymptotic branch.
} 


\section{The single-junction-switch versus -flip dynamics}

Single-junction-flip Metropolis generation series came also, by applying the same annealing schedule scheme, to equilibria. Nevertheless, the single-junction-flip Metropolis generation series contrast with the single-junction-switch Metropolis generation series into two major ways:

(i) The total amounts of surprisal at single-junction-flip equilibria within the realworld window 2-3 (Newman 2018) appear to be greater than the total amounts of surprisal of the self-fit outputs by about 300 times their standard-deviations-while the corresponding ones at single-junction-switch equilibria are greater by at most 8 times their standard-deviations. This makes the single-junction-flip Metropolis algorithm clearly inconsistent with the self-fit join principle, hence unrealistic.

(ii) For large effective scaling exponent $\widetilde{\lambda}$ values, the single-junction-flip simulations appear much less subject to noise.

In brief: restricting matchings to maximal matchings renders our system realistic but numerically unstable for relatively large scaling exponents; vice versa, loosing matchings renders our system unrealistic but numerically stable for a relatively wider range of scaling exponents.

To explain this, we must keep in mind that our particular working assumptions neglect junctions. In fact, in one hand, the single-junction-switch dynamics provides to our working assumptions a "hard-coded" constraint on junction layouts so that our system becomes more realistic. On the other hand, the single-junction-flip dynamics allows the Metropolis algorithm to reject maximal matchings in favour of non-maximal matchings so that our algorithm becomes numerically more stable. To resolve this dilemma, we may replace the hard constraint on junction layouts with a soft constraint. This may take, in the total amount of surprisal $S_{\mu}$ (28), the form of additional surprisal terms involving junctions or mixing streets and junctions. The derivation of such terms is however outside the scope of the present paper.

\section{Unorthodox Watts-Strogatz phase diagram}

So far we have demonstrated that Central London sustains scale-free configurations of streets over a wide range of scalings. This means that the selection of a realistic scalefree configuration of street involves other criteria than just scale-freeness. An appealing explanation might hold with the small-world crossover, which may happen as we exposed in our "A brief comparison with Ising models" section. This hypothesis illustrates well the new class of explorations that the method presented in the present paper brings in the field. We keep our hypothesis for future work. Meanwhile, to emphasize our contribution, we demonstrate the small-world crossover by adopting the phase diagram used for Watts-Strogatz small-world models (Watts and Strogatz 1998; Newman 2003, 2018).

\section{Two-regime phase diagram}

The Watts-Strogatz phase diagram for Central London plotted in Fig. 8 shows two crossovers which occurs simultaneously at the effective scaling value of 3 . This phase diagram plots for the information networks of Central London the averages of their 


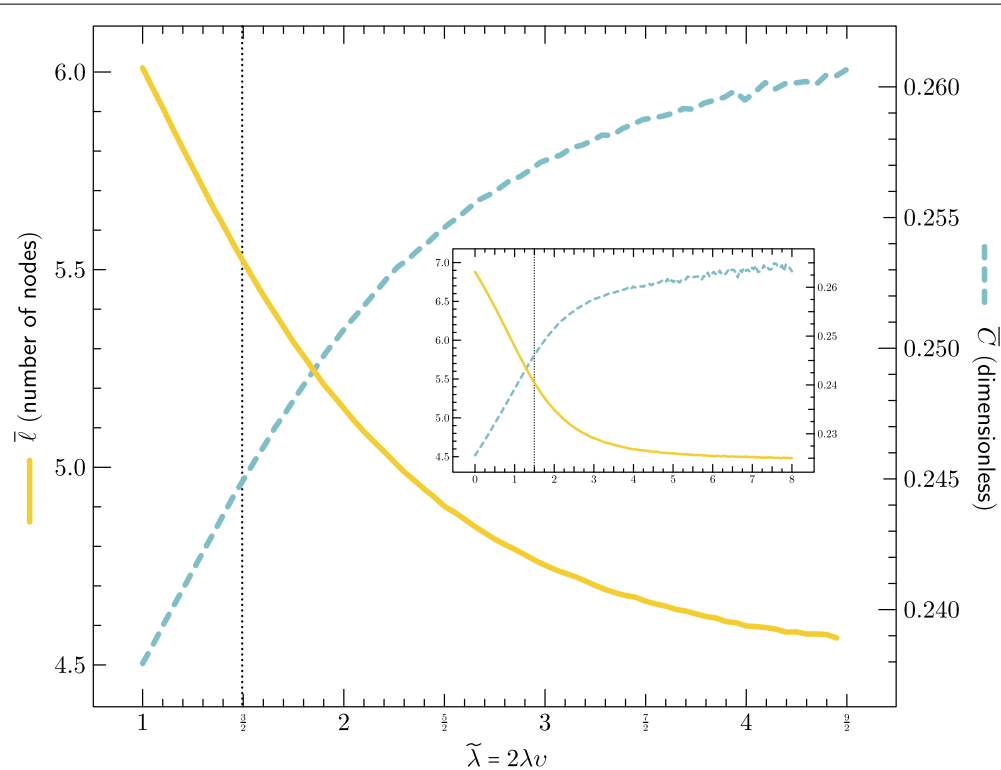

Fig. 8 Mean geodesic distance between nodes and mean local transitivity versus effective scaling exponent for the information networks of Central London (United Kingdom): yellow solid line plots the mean of the mean geodesic distance between node pairs $\ell$; turquoise dashed line plots the mean of the mean local transitivity $C_{\text {; }}$ the vertical dotted line represents the effective scaling exponent $\widetilde{\lambda}_{0}$ for which the associated Metropolis equilibrium approaches on average self-fit outputs — as illustrated in Fig. $6 \mathrm{~d}$; the inset shows their asymptotic behaviours; the main figure and the inset have the same aspect ratio. The experimental protocol was the same as in Fig. 7. (We attribute the noise to the same reasons as in Fig. 7.)

mean geodesic distance (or mean vertex-vertex distance) $\ell$ and of their mean local transitivity (or clustering coefficient) $C$ as functions of the rewiring parameter (Watts and Strogatz 1998; Newman 2018). The rewiring parameter is here the effective scaling exponent $\tilde{\lambda}$. These two functions experience a qualitative change of behaviour in the vicinity of effective scaling $\widetilde{\lambda}=3$, that is, they exhibit a crossover at effective scaling $\widetilde{\lambda}=3$ (Gluzman and Yukalov 1998). Our claim that the crossovers precisely happen at effective scaling $\widetilde{\lambda}=3$ relies on the arguments given by Cohen and Havlin (2003). The two simultaneous crossovers indicate two distinct phases or regimes:

(i) A uniform regime takes place as effective scaling increases from 3. As effective scaling gets higher and higher starting from 3 , the mean geodesic distance between node pairs $\ell$ (resp. the mean local transitivity $C$ ) tends on average asymptotically towards a slightly-decreasing (resp. a slightly-increasing) plateau. The asymptotic behaviours become obvious around the effective scaling value of 4 . This means that in this regime the involving phenomena are saturating.

(ii) An emergent/reduction regime occurs as effective scaling decreases from 3. As effective scaling gets lower and lower starting from 3, the mean geodesic distance between node pairs $\ell$ (resp. the mean local transitivity $C$ ) increases (resp. decreases) on average to reach a linear behaviour around the effective scaling value of $3 / 2$. The decreasing on average of the mean geodesic distance between node pairs $\ell$ as scaling increases means that scaling is inducing a smaller world (Newman 2018). The increasing on average of the mean local transitivity $C$ as scaling increases means that scaling is inducing a denser world or a world with less "struc- 
tural holes" (Newman 2018). In our context, a smaller world means journeys with lesser changes of streets ( see end of point (iv) in "A brief comparison with Ising models" section); a denser world means more local alternative routes.

We may regard the two linear behaviours for small and large scalings as degenerate or extreme. In this sense the relevant part of the phase diagram yields between the effective scaling values of $3 / 2$ and 4 . This is consistent with our previous expectation in "Equilibria" section that the relevant physics of our system may occur within the window 1-4.

\section{A brief comparison with the classical Watts-Strogatz phase diagram}

The Watts-Strogatz phase diagram for Central London confirms that our urban street network model underlies the small-world effect. Our expectation was sketched in point (iv) of our "A brief comparison with Ising models" section. Nonetheless the obtained Watts-Strogatz phase diagram differs from the classical Watts-Strogatz phase diagram (Newman 2003, Fig. 6.2; Watts and Strogatz 1998, Fig. 2) in three essential characteristics:

(i) A smaller world means a denser world, not a less dense one. This is because on average the mean local transitivity $C$ increases instead of decreasing.

(ii) The two crossovers coincide. In other words, no emergent/reduction regime overlaps with an uniform regime and vice versa.

(iii) The small-world effect predominates. The overall variation of the average of the mean local transitivity $C$ is of order 0.04 , so we may regard the local transitivity evolution as insignificant. Meanwhile the average of the mean geodesic distance between node pairs $\ell$ gains overall 2.5 nodes and 0.9 nodes within the relevant window from $3 / 2$ to 4 , that is, the small-world effect is actually the substantial phenomenon.

Therefore, contrary to Watts-Strogatz small-world networks (Watts and Strogatz 1998; Newman 2003, 2018), the information networks of Central London experience no balance between local transitivity and the small-world effect. Actually, among both, only the small-world effect is relevant as scaling varies.

\section{The self-fit configurations of streets are inefficient}

Generation series (d) in Fig. 6 shows that, for Central London, self-fit outputs are hardly distinguishable from Metropolis in-equilibrium generations at effective scaling $\widetilde{\lambda}_{0}=1.495$. This value can be regarded as a measurement of the effective scaling at which Central London sustains self-fit configurations of streets. This measurement is represented on the Watts-Strogatz phase diagram for Central London in Fig. 8 by the vertical dotted line. The phase diagram immediately tells us on self-fit information networks for Central London three noteworthy facts:

(i) Their worlds are on average of a magnitude one node larger. As natural reference, we take here the high-scaling asymptotic configurations of streets.

(ii) They occur around the end of the low-scaling linear behaviour. That is, they occur around the low-scaling boundary of the relevant window 3/2-4. 
(iii) There is room for information networks with significantly smaller worlds. A quick check shows that information network worlds at effective scaling 2.50 (centre of realistic window 2-3) and 2.75 (centre of the relevant window 3/2-4) are on average, respectively, 0.6 and 0.7 nodes smaller than the self-fit worlds. These offset drops are, respectively, of the order of $60 \%$ and $70 \%$. Namely, they are substantial.

To summarize, the Watts-Strogatz phase diagram describes self-fit information networks for Central London as being on average relatively large worlds.

However, Central London dwellers may rather want to know whether their self-fit configurations of streets are efficient. Efficiency means here for city-dwellers that they can complete their journeys as fast as possible. This can be partially achieved by decreasing as much as possible the number of street changes required per journey. This means to develop information network whose worlds are as small as possible. This corresponds on the Watts-Strogatz phase diagram to information networks having on average relatively small worlds. In effect, this involves the information networks that actually experience the crossover. As we have seen, quite the opposite actually happens to the self-fit information networks of Central London: they take place where the small-scaling linear behaviour ceases and they underlie on average relatively large worlds. In brief, the self-fit configurations of streets for Central London are inefficient.

\section{Conclusions and future works}

Unplanned or self-organized urban street networks undergo a scale-free coherence that we interpret in terms of a fluctuating system. This paper sketches how the Metropolis algorithm, which embodies well the idea of fluctuating systems (Newman and Barkema 1999; Landau and Binder 2015), can apply to self-organized urban street networks once our interpretation is embraced. The Metropolis algorithm is a classical entry-point for more elaborate Monte Carlo methods. These methods are the natural numerical companions for theoretical studies on fluctuating systems, and vice versa. Our theoretical framework is the maximum entropy formalism (MAxENT) (Jaynes 2003; Grandy 1987; Lawrence 2019).

Our prior hypothesis (Jaynes 2003; Grandy 1987) is scale-freeness (Stanley 1971). Assuming this property as the result of an underlying self-similarity symmetry (Mandelbrot 1982; Batty 2008) paves the way to a symmetry-conservation correspondence as used in physics (Gross 1996; Romero-Maltrana 2015). This physical idea effortlessly adapts itself to MaxEnT. This allows us, as required for implementing any Monte Carlo method, to set up our prescribed statistical equilibrium. The self-similarity symmetry demands the conservation on average of the logarithm of an extensive quantity which, by virtue of MaxEnt, most plausibly underlies a discrete Pareto distribution (Clauset et al 2009). The scaling exponent is our equilibrium parameter. Meanwhile, the best we can tell on any information network is that it is a mesoscopic system whose objects, nodes (streets) and edges (junctions), have equiprobable configurations. So, the best we can assume about our extensive quantity is that it is a number of equiprobable configurations. The conserved quantity becomes then an average of Boltzmann entropies. 
However we may rather interpret this information measure as an amount of surprisal (Tribus 1961; MacKay 2003; Applebaum 2008; Stone 2015; Lawrence 2019) that actually quantifies the comprehension of the city-dwellers for their own urban street network (Benoit and Jabari 2019a, b). Once our prescribed statistical equilibrium is fully set up, we can readily implement our Metropolis acceptance ratio.

As concerns the ergodic dynamics, its counterpart, the nonoverlapping walk approaches found in the literature (Porta et al 2006; Jiang et al 2008; Viana et al 2013) appear inappropriate but nonetheless inspirational. We imagine information networks not in terms of haphazard nonoverlapping walks along street-segments, but in term of random street layout at junctions. Our approach readily leads to dynamics that mimic the classical single-spin-flip dynamics in Ising models (Newman and Barkema 1999; Landau and Binder 2015; Berlinsky and Harris 2019). At every junction, each pair of street-segments that can hold a street is a link of a graph where street-segments map to nodes, so that each matching (Pemmaraju and Skiena 2003) of this graph represents a possible layout. As the single-spin-flip dynamics changes the state of a spin into another possible state, our dynamics changes the matching (layout) of a junction into another possible matching (layout). We named single-junction-flip the dynamics that involves any matchings, and single-junction-switch the dynamics that involves only maximal matchings (Pemmaraju and Skiena 2003). If our approach implicitly implies that selforganized urban street networks might sustain scaling coherence over a wide range of scalings, finding dynamics reminiscent of Ising models suggests first and foremost that they might undergo a crossover as scaling varies. Since large scale-free networks exhibit ultra-small- and small-world behaviours for scaling values respectively smaller and greater that 3 (Cohen and Havlin 2003), self-organized urban street networks might actually experience as scaling increases a small-world crossover around the scaling value of 3.

We choose as case study the recognized self-organized urban street network of Central London (United Kingdom) (Jacobs 1993). Simulations based on predominant streets and an asymptotic agent model driven by social interactions (Dover 2004; Benoit and Jabari 2019a, b) show that the single-junction-switch Metropolis algorithm generates equilibria that are consistent with the aforementioned nonoverlapping walk approaches. The simulations remain consistent over a range of scaling exponents large enough to contain the realistic window from 2 to 3 (Newman 2018) and to capture changes of behaviour in their total and average amounts of surprisal. Thusly, the single-junction-switch Metropolis algorithm allows simulational investigations. The single-junction-flip dynamics also leads to equilibria, but with unrealistic amounts of surprisal. We explain this, given that our model neglects junctions while the single-junction-switch dynamics coerces junctions to have maximal layouts, by a lack of constraints on junctions. Along this explanation, the single-junction-flip dynamics may allow to investigate the role played by junctions. In brief, our simulations on Central London show that our adaptation of the Metropolis algorithm for generating self-organized information networks is applicable and relevant.

To illustrate our innovative methodology, we plot the Watts-Strogatz phase diagram with scaling as rewiring parameter. The phase diagram exhibits an emergent/reduction 
regime followed by an uniform regime as scaling increases. That is, the small-world and the local transitivity crossovers occur simultaneously. However only the former is significant in magnitude. The crossovers happen approximately around the scaling value of 3. More noticeably, the crossovers curve within the realistic window from 2 to 3 . Thusly, as expected, our phase diagram demonstrates a small-world crossover around the scaling value of 3. Our phase diagram also allows us to discuss the pertinence of the self-fit outputs. The self-fit outputs take place on average nearly the scaling value of $3 / 2$, namely, significantly before the realistic window 2-3. They actually occur on average at the ending of the linear scaling behaviour observed at low scalings-which we may consider as degenerate. Concretely this means that self-fit outputs generate on average information networks that underlie relatively large worlds, namely, that are inefficient. If the implicit belief that self-organized urban street networks have reached an optimal balance over time holds, representative information networks may rather occur within the realistic window 2-3 where their worlds are on average relatively small, namely, efficientassuming that the small-world effect gets counterbalanced as its effect curves. Thusly, our illustrative Watts-Strogatz phase diagram challenges the state-of-the-art on generating information networks, while it indicates that self-organized information networks can undergo as scaling increases a small-world crossover curving within the realistic window 2-3. In other words, our illustrative numerical "experiment" on Central London demonstrates that our adaptation of the Metropolis algorithm for generating self-organized information networks is indeed pertinent to gain new insights.

From a fundamental point of view, future works must focus on two points. First, we must recognize the deep origin underlying the extensive quantity associated to the scaling exponent in order to specify its very nature. Second, we must find an uncoercive way to involve junctions in order to investigate their role. From a simulational point of view, we must investigate the undergoing small-world crossover by considering other network phenomena (Newman 2018) and a large panel of recognized self-organized urban street networks (Jacobs 1993; Crucitti et al 2006). We anticipate to observe network phenomena (Newman 2018) that counterbalance the small-world effect within or around the realistic window 2-3 (Newman 2018). From an observational point of view, our fluctuating approach clearly challenges the current method to determine the scaling exponent of an urban street network which is based on a single arbitrary output (Porta et al 2006; Jiang et al 2008). In view to confront our simulational data against observational data, we must derive methods able to "take" the scaling exponent and to measure network measures (Newman 2018) along with their susceptibilities (Newman and Barkema 1999; Grandy 1987; Jaynes 2003). From a practical point of view, we envision that our Metropolis adaptation may initiate, alongside Monte Carlo models getting more elaborate but also more realistic, a 'scaling-dynamics' based description of our urban street networks and, by extension, of our cities. Such a descriptive framework may provide fruitful analogies with thermodynamics and precious insights on unplanned evolution for city scientists, city designers, and decision-makers to anticipate the evolution of our cities. 


\section{Supplementary Information}

The online version contains supplementary material available at https://doi.org/10.1007/s41109-021-00375-6.

Additional file 1. State-of-the-art construction paradigm for configurations of streets (Animation A1)

Additional file 2. Single-junction-switch Metropolis algorithm for configurations of streets (Animation A2).

\section{Acknowledgements}

This work was supported by the NYUAD Center for Interacting Urban Networks (CITIES), funded by Tamkeen under the NYUAD Research Institute Award CG001 and by the Swiss Re Institute under the Quantum Cities ${ }^{\text {TM }}$ initiative.

\section{Authors' contributions}

JGMB conceived and designed the study, collected and treated the map data, designed and programmed the simulation tools, performed and treated the simulations, and wrote the manuscript. SEGJ helped to shape the manuscript. Both authors read and approved the final manuscript.

\section{Availability of data and materials}

The map of the urban street network of Central London was extracted from the Open Street Map (OSM) comprehensive archive (OpenStreetMap 2004-2020) and simplified with OSMnx V0.11.4 (Boeing 2017). The network measures were computed with the igraph C library v0.8.2 (Csárdi and Nepusz 2006; Csárdi et al 2020). The software used to perform the simulations is available, along with the map and generation series samples, at https://doi.org/10.5281/ zenodo.3746140. The datasets generated and analysed during the current study are available from the corresponding author on reasonable request.

\section{Declarations}

Competing interests

The authors declare that they have no competing interests.

\section{Author details}

${ }^{1}$ New York University Abu Dhabi, Saadiyat Island, POB 129188, Abu Dhabi, UAE. ${ }^{2}$ Ronin Institute, Montclair, NJ 07043, USA.

${ }^{3}$ New York University Tandon School of Engineering, Brooklyn, NY 11201, USA.

Received: 23 April 2020 Accepted: 14 April 2021

Published online: 29 April 2021

\section{References}

Aczél J (1966) Lectures on functional equations and their applications, mathematics in science and engineering, vol 19. Academic Press, New York. https://doi.org/10.1016/S0076-5392(09)60202-2

Applebaum D (2008) Probability and information: an integrated approach, 2nd edn. Cambridge University Press, Cambridge. https://doi.org/10.1017/CBO9780511755262

Batty M (2008) The size, scale, and shape of cities. Science 319(5864):769-771. https://doi.org/10.1126/science.1151419

Benoit J, Jabari S (2019) On the perturbation of self-organized urban street networks. Appl Netw Sci 4:49. https://doi.org/ 10.1007/s41109-019-0153-0

Benoit J, Jabari S (2019) Structure entropy, self-organization and power laws in urban street networks. arXiv:abs/1902. 07663

Berlinsky AJ, Harris AB (2019) Statistical mechanics. Graduate Texts in Physics, Springer, Cham. https://doi.org/10.1007/ 978-3-030-28187-8

Boeing G (2017) OSMnx: new methods for acquiring, constructing, analyzing, and visualizing complex street networks. Comput Environ Urban Syst 65:126-139. https://doi.org/10.1016/j.compenvurbsys.2017.05.004

Clauset A, Shalizi CR, Newman MEJ (2009) Power-law distributions in empirical data. SIAM Rev 51(4):661-703. https://doi. org/10.1137/070710111

Cohen R, Havlin S (2003) Scale-free networks are ultrasmall. Phys Rev Lett 90(5):058701. https://doi.org/10.1103/PhysR evLett.90.058701

Crucitti P, Latora V, Porta S (2006) Centrality measures in spatial networks of urban streets. Phys Rev E 73(3):036125. https://doi.org/10.1103/PhysRevE.73.036125

Csárdi G, Nepusz T (2006) The igraph software package for complex network research. Int J Complex Syst 1695(5):1-9

Csárdi G, Nepusz T, Horvát S, Traag VA, Zanini F (2020) igraph. https://doi.org/10.5281/zenodo.3774399

Dover Y (2004) A short account of a connection of power laws to the information entropy. Physica A 334(3-4):591-599. https://doi.org/10.1016/j.physa.2003.09.029

Galassi M, Davies J, Theiler J, Gough B, Jungman G, Alken P, Booth M, Rossi F (2009) GNU scientific library reference manual, 3rd edn. Network Theory Limited, https://www.worldcat.org/isbn/9780954612078

García-Pérez G, Boguñá M, Serrano MÁ (2018) Multiscale unfolding of real networks by geometric renormalization. Nat Phys 14(6):583-589. https://doi.org/10.1038/s41567-018-0072-5

Gluzman S, Yukalov VI (1998) Unified approach to crossover phenomena. Phys Rev E 58(4):4197-4209. https://doi.org/10. 1103/PhysRevE.58.4197 
Grandy WT Jr (1987) Foundations of statistical mechanics, fundamental theories of physics, vol 19. Springer, Dordrecht. https://doi.org/10.1007/978-94-009-3867-0

Gross DJ (1996) The role of symmetry in fundamental physics. Proc Natl Acad Sci USA 93(25):14256-14259. https://doi. org/10.1073/pnas.93.25.14256

Hosoya $\mathrm{H}$ (1971) Topological index. A newly proposed quantity characterizing the topological nature of structural isomers of saturated hydrocarbons. Bull Chem Soc Jpn 44(9):2332-2339. https://doi.org/10.1246/bcsj.44.2332

Jacobs AB (1993) Great streets. The MIT Press, Cambridge

Jaynes ET (1957) Information theory and statistical mechanics. Phys Rev 106(4):620-630. https://doi.org/10.1103/PhysR ev.106.620

Jaynes ET (2003) Probability theory: the logic of science. Cambridge University Press, Cambridge. https://doi.org/10.1017/ CBO9780511790423

Jiang B, Claramunt C (2004) Topological analysis of urban street networks. Environ Plan B 31(1):151-162. https://doi.org/ $10.1068 / \mathrm{b} 306$

Jiang B, Zhao S, Yin J (2008) Self-organized natural roads for predicting traffic flow: a sensitivity study. J Stat Mech Theor Exp 7:P07008. https://doi.org/10.1088/1742-5468/2008/07/P07008

Kalapala V, Sanwalani V, Clauset A, Moore C (2006) Scale invariance in road networks. Phys Rev E 73(2):026130. https://doi. org/10.1103/PhysRevE.73.026130

Knuth DE (1997) Seminumerical algorithms, the art of computer programming, vol 2, 3rd edn. Addison-Wesley, Reading

Landau DP, Binder K (2015) A Guide to Monte Carlo simulations in statistical physics, 4th edn. Cambridge University Press, Cambridge. https://doi.org/10.1017/CBO9781139696463

Lawrence A (2019) Probability in physics. Undergraduate Lecture notes in physics. Springer, Cham. https://doi.org/10. 1007/978-3-030-04544-9

MacKay DJC (2003) Information theory, inference, and learning algorithms. Cambridge University Press, Cambridge. https://doi.org/10.5555/971143

Mandelbrot BB (1982) The fractal geometry of nature. W. H. Freeman and Company, New York

Masucci AP, Stanilov K, Batty M (2014) Exploring the evolution of London's street network in the information space: a dual approach. Phys Rev E 89(1):012805. https://doi.org/10.1103/PhysRevE.89.012805

Metropolis N, Rosenbluth AW, Rosenbluth MN, Teller AH, Teller E (1953) Equation of state calculations by fast computing machines. J Chem Phys 21(6):1087-1092. https://doi.org/10.1063/1.1699114

Molinero C, Murcio R, Arcaut E (2017) The angular nature of road networks. Sci Rep 7(1):4312. https://doi.org/10.1038/ s41598-017-04477-z

Newman MEJ (2003) The structure and function of complex networks. SIAM Rev 45(2):167-256. https://doi.org/10.1137/ S003614450342480

Newman MEJ (2018) Networks: an introduction, 2nd edn. Oxford University Press, Oxford. https://doi.org/10.1093/oso/ 9780198805090.001 .0001

Newman MEJ, Barkema GT (1999) Monte Carlo methods in statistical physics. Oxford University Press, Oxford

OpenStreetMap (2004-2020) OpenStreetMap Project. https://www.openstreetmap.org

Pemmaraju S, Skiena S (2003) Computational discrete mathematics: combinatorics and graph theory with mathematica. Cambridge University Press, Cambrige. https://doi.org/10.1017/CBO9781139164849

Porta S, Crucitti P, Latora V (2006) The network analysis of urban streets: a dual approach. Physica A 369(2):853-866. https://doi.org/10.1016/j.physa.2005.12.063

Press WH, Teukolsky SA, Vetterling WT, Flannery BP (2007) Numerical recipes: the art of scientific computing, 3rd edn. Cambridge University Press, Cambridge

Romero-Maltrana D (2015) Symmetries as by-products of conserved quantities. Stud Hist Philos Sci B 52:358-368. https:// doi.org/10.1016/j.shpsb.2015.10.006

Rosvall M, Trusina A, Minnhagen P, Sneppen K (2005) Networks and cities: an information perspective. Phys Rev Lett 94(2):028701. https://doi.org/10.1103/PhysRevLett.94.028701

Rybski D, Arcaute E, Batty M (2019) Urban scaling laws. Environ Plan B 46(9):1605-1610. https://doi.org/10.1177/23998 08319886125

Stanley HE (1971) Introduction to phase transitions and critical phenomena, international series of monographs on physics, vol 46. Oxford University Press, London

Stone JV (2015) Information theory: a tutorial introduction. Sebtel Press, Sheffield. https://doi.org/10.13140/2.1.1633.8240

Tribus M (1961) Thermostatics and Thermodynamics. University Series in Basic Engineering, Van Nostrand, Princeton https://hdl.handle.net/2027/mdp.39015001333361

Viana MP, Strano E, Bordin P, Barthélemy M (2013) The simplicity of planar networks. Sci Rep 3:3495. https://doi.org/10. 1038/srep03495

Watts DJ, Strogatz SH (1998) Collective dynamics of'small-world' netwoks. Nature 393(6684):440-442. https://doi.org/10. 1038/30918

\section{Publisher's Note}

Springer Nature remains neutral with regard to jurisdictional claims in published maps and institutional affiliations. 